\title{
Reminiscences
}

\author{
Brigitte Schmieder
}

(C) Springer $\bullet \bullet \bullet \bullet$

\begin{abstract}
I would like to thank Solar Physics colleagues for asking me to write this chapter on my professional life. My main interest has always been focused on the Sun, our star, from the heating of the corona, to the dynamics of prominences and their eruptions, flares and coronal mass ejections until their impact on the Earth. I built a new group in solar physics and gave to them my enthusiasm. They brought to me a lot of satisfaction. We have made important advances in solar physics with a step forward to understand the triggers of solar activity and their terrestrial effects. Our avant-garde research and discovery has opened new topics for the solar community. Mixing observations obtained on the ground and in space with theory and numerical simulations brings a new perspective in research.
\end{abstract}

\section{Early days}

\subsection{Chilhood}

I grew up after the second world war in an intellectual family with my older sister. My mother always motivated us to learn very hard and get a good job. She had been a little frustrated by not being able to do what she wanted because of the war. She travelled before her wedding to Greece and Algeria alone. It was each time an adventure for her. My father was an engineer who worked in a shipping company when he was single, landing in famous harbors in Asia (Yokohama, Hong Kong, and Saigon). Just before the war, he was hired by an oil company but was rapidly appointed to do charcoal work in a forest in Burgundy. Therefore we did not go to school and my mother was our teacher before we passed the exam at age ten to be accepted into the Lycée Marie Curie in Sceaux (city located $7 \mathrm{~km}$ to the South of Paris). My childhood was very peaceful (Figure 1 top left panel). After literature and Latin, I studied mathematics and physics with Catherine Lacombe and after the baccalaureate I was accepted in the Lycée Fénelon in Paris. After studying mathematics with Prof. Dixmier and mechanic of fluids with Prof. Germain at the Sorbonne I attended the lectures

1 Observatoire de Paris, LESIA, 5 Place Janssen, 92195

Meudon, France email: brigitte.schmieder@obspm.fr ${ }^{2}$ PSL

Research University, CNRS Sorbonne Université, Univ. Paris

06, Univ. Paris Diderot, Sorbonne Paris Cité 

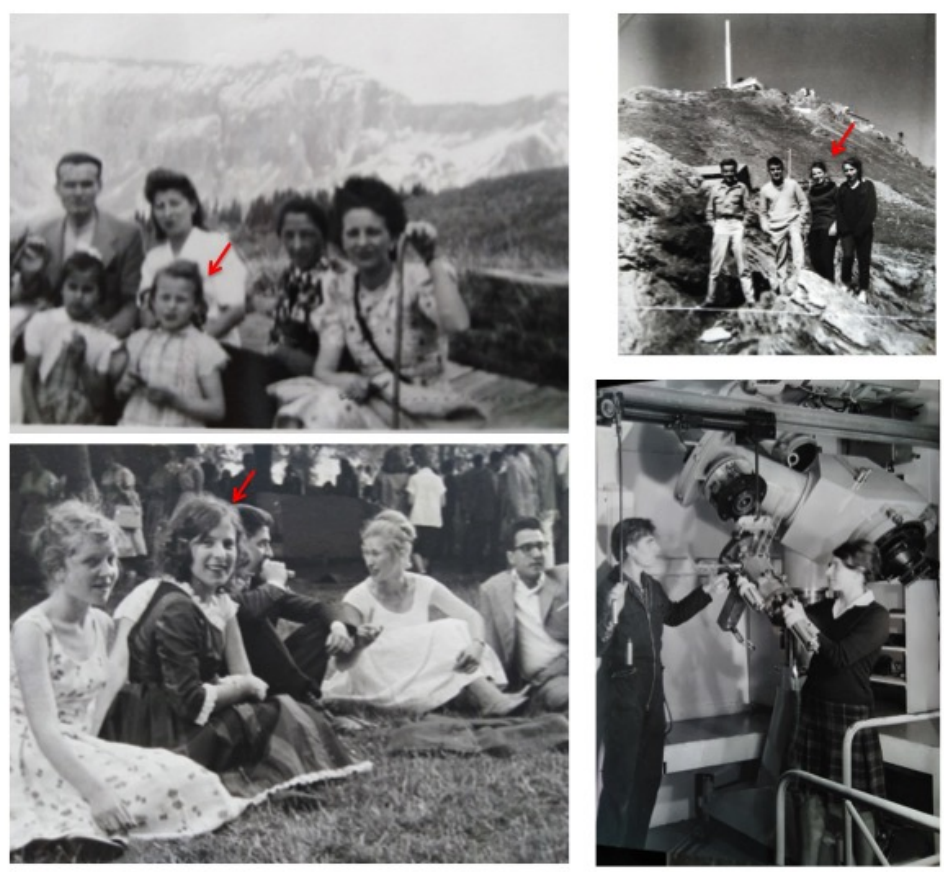

Figure 1. Top left, with my sister, my father, my mother (M. and Mme. Pailhas) and two of their friends in Combloux (Alps), top right: with the student group of G.Wlérick at Pic du Midi, bottom left: high school class-mates, bottom right: Saint Michel l'Observatoire, with the electronic camera at the focus plan of the $193 \mathrm{~cm}$ mirror telescope. I am indicated by red arrows.

of astrophysics with Profs. Evry Schatzman (the greatest physicist whom I ever met), Jean-Claude Pecker, Jean Paul Zahn, Henri van Regemorter, Roger Cayrel and Gérard Wlérick. During this time I belonged to a group of students and young post docs, doing sport, skiing and tennis (Figure 1 bottom left panel). We were meeting every Friday in a café in the Sorbonne square, "chez Sylvain" to drink coffee and to decide what we would do the next week end. Ngyuen Rieu belonged to the group and spoke to me about astrophysics. I was fascinated by this kind of research and by the Observatoire in Meudon where he had a position in the radio astronomy department. Therefore when G. Wlérick asked if students were interested in training, I followed him and went to Meudon once a week. When I entered his office for the first time, he told me that my name "Pailhas" was familiar to him because his father, a disciple of Rodin, sculpted the bust of Mrs. Pailhas when she was very young. In fact, she was the wife of my grand uncle.

After the master degree in astrophysics, I started to work at the Observatory of Paris in Meudon (in the stables of the castle) with G. Wlérick preparing for a "Doctorat es Sciences". The research for my thesis effectively started after 1968, the year of the revolution of French students when Pierre Mein, my thesis advisor moved from the "Institut d'Astrophysique" ( IAP) in Paris to Meudon in the solar department. I defended my thesis in 1977. 


\subsection{Training time}

During this long time period with G. Wlérik and P. Mein, I learned astrophyiscs in many of its different aspects. My training started by looking at the spectra obtained during the eclipse of 1961 at the "Observatoire de Haute Provence" in Saint Michel. I discovered that G.Wlérick gave this task to each student who arrived in his laboratory. Then, I computed theoretically the ratio of the forbidden Ni XV lines using atomic data updated by Burgess and Seaton in the UK. I wanted to compare my results with the spectral observations of Bernard Lyot obtained at Pic du Midi. One morning Audouin Dollfus brought me the boxes containing the frames made by B. Lyot. One frame was missing and it was just the frame of the wavelengths of the Ni XV. We never found it. During this time I was also working with the electronic camera invented and developed by André Lallemand in a laboratory in Paris. We had to check the cells and had to make a vacuum in the tube during half a day before observing. With the group of students of G. Wlérick we observed the planets (using UBV filters) at Pic du Midi and at the "Observatoire de Haute Provence" with the new $193 \mathrm{~cm}$ telescope (Figure 1 right panels). It was really fun to wait during the night for a clear sky. One winter we built an igloo in the snow on the terrace of the Pic du Midi Observatory during our waiting periods. I remember a few of my colleagues: Pierre Charvin, Loic Vapillon, Michel Combes, Paul Felenbok, Philippe Véron, and Michelle Loulergue and many young female students from the "Ecole Normale Supérieure" of Fontenay-aux-Roses.

Back in Meudon, I had to work to build a new spectrograph in a building called "Petit Siderostat" with the engineers Rolland Hellier and Christian Coutard. It never really worked because we put a selector of wavelengths after the grating which could not be adjustable. After that I participated with R.Michard in the construction of the solar tower telescope implemented by the first Multichannel Substrative Double Pass (MSDP) spectrograph invented by Pierre Mein. The observatory in Meudon developed quickly during these years and after the construction of the solar tower we moved to new buildings adjacent to the tower. During this time, I acquired an extensive knowledge in instrumentation, in astrophysics and in atomic physics. All these different works did not lead to any publication but they would be very helpful for my future work in science. However, I realized only much later than they did not help me to get a better position. I learned at my own expense that research not published is not valuable. It is why now I always push my students and collaborators to publish their results and I am well known to ask them frequently "Where is the paper?".

\subsection{Heating of the corona -Thesis- 70 's}

When Pierre Mein arrived in Meudon, I finally got a topic for my thesis: "Solar coronal heating by dissipation of acoustic waves: development of a radiative code and applications to observations".

In 1948 Evry Schatzmann proposed that acoustic wave dissipation could work for heating the corona. Grant Athay, an American researcher confirmed this hypothesis after theoretical computations that such a mechanism could be efficient. In fact nobody was really measuring the phases, velocities and amplitudes of the waves. With my observations we concluded that the waves were reflected back in the transition region between the chromosphere and the corona and were mainly standing waves. They could not heat the corona. The dissipation was too small. Before 1981 I published seven papers either alone or with Nicole Mein on this topic Schmieder, 1972, Schmieder, 1976; Schmieder, 1977, Schmieder, 1978, Schmieder, 1979, Schmieder and Mein, 1980 
Mein and Schmieder, 1981). After my thesis, Christensen Dalsgaard told me that I had to change my radiative transfer code in LTE (local thermodynamic equilibrium) from 2 to 3 dimensions because we had discovered that the oscillations were resonant waves in the 3D sphere of the Sun. I would have to transform my full box of computer punch cards with small holes to a new code. Each day I had to transport this box to Orsay where the computer ran the program overnight. Often I came back the next day to discover that I had forgotten a comma somewhere. It was too tiring to run such a heavy code. Therefore I decided to change of topic after my thesis defense (see Section 2.1).

About the observations concerning the waves, it had been also a challenge to get them. The solar tower in Meudon was just built and the spectrograph allowed us to get spectra of the $\mathrm{Mg} \mathrm{I} \mathrm{b}$ line in the low chromosphere, as well as $\mathrm{H} \alpha$ and $\mathrm{Ca} \mathrm{K}$ lines in the chromosphere. After the observations, I was running a Fourier transform code and discovered that I was measuring the oscillations of the solar tower, or of people who were going down the stairs. Indeed Jean Claude Pecker and the engineers had an office upstairs. The interior and the external towers were tied together in the basement by concrete. After inspection, M. Remondet, the architect, agreed to separate the two towers with a hammer drill. At the same time Pierre Mein thought that the best way to track the granules and the chromospheric pattern was to open the slit and use computations to follow them. It was the birth of the MSDP. In fact Pierre Mein spent one year at Sacramento Peak Observatory in New Mexico and the best for me was to go there (my first airplane trip lasted 36 hours!). I observed there during the day and digitised the spectra during the night. They had great facilities that we did not have in France at that time. I met many famous scientists in Sac Peak: Dave Rust, Richard Altrock, Richard Canfield, and only one woman Joan Vorpahl. When Pierre Mein was at Sac Peak and I was back in Meudon, I could discuss my problems of radiative transfer with Philippe Delache and Christian Magnan.

I had to develop every thing by myself with no existing material, e.g., codes or observations. Pierre Mein was there to encourage me and gave me good advice every six months. I would like really to thank him for his bright ideas. Pierre Mein and Nicole Mein are still working on the new generation of MSDP and very active to observe, enthusiastic to take part in coordinated campaigns. I had and still have an intensive collaboration with them throughout my research career (see my recent paper on prominence-tornadoes with them using observations made at the Meudon solar tower with the MSDP (Schmieder et al., 2017b)).

\subsection{Family}

During my thesis I had also another task to do. A thesis was at that time a long piece of work and could last for ten years before the change of thesis format to fit with the international $\mathrm{PhD}$ thesis of 3 years. I built a family with three children: one boy Laurent and two daughters Anne and Céline (Figure 2). I would like to thank my husband Eric who always helped me to realize my wishes, to go to Sac Peak for one month with a baby of two years at home. I could manage the mother's life with my work thanks to him. After bringing the children to school at 8:30 am I was back at 6 pm for the homework.

My husband after his university studies, his administration school ("Ecole Nationale d'administration" - ENA) and his military service entered in the Ministry of health directed by Simone Veil. He was hard working and came back home only after 8 or 9 $\mathrm{pm}$. We were well organized with a nurse who received nearly all my salary. But I knew that it would benefit me in the future. This situation lasted only during 15 years. It 

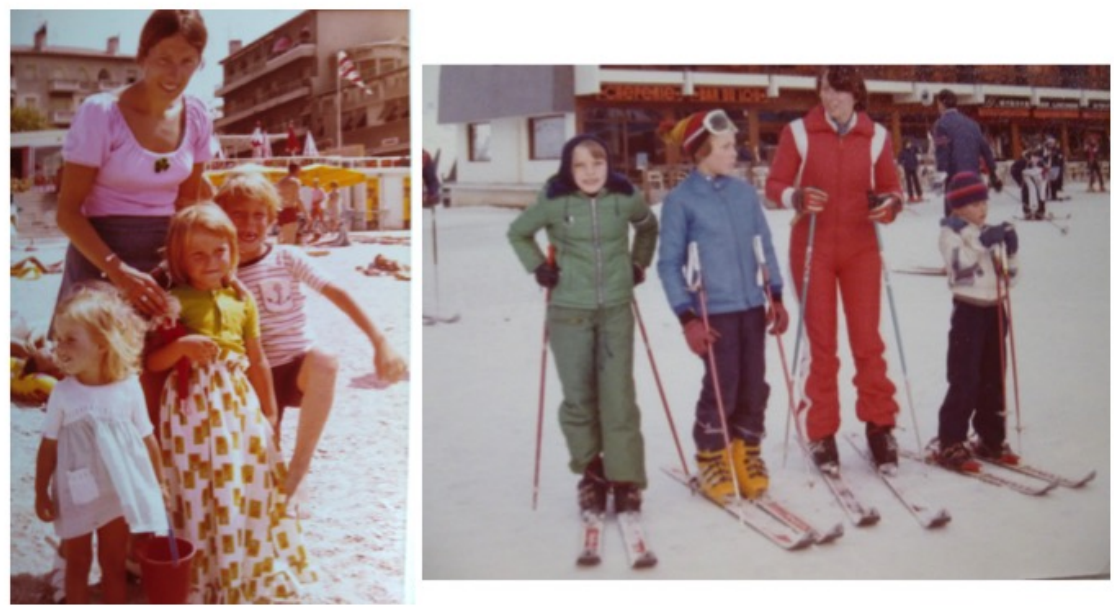

Figure 2. Left, on the French riviera with my 3 children: Céline, Anne and Laurent, right, winter sport in the Alps with Anne, Laurent and Céline.

is a short period of time in a long professional career. I never interrupted my career for more than 6 weeks even with my three maternity leaves. Research is progressing fast and we always need to read and update our knowledge to be able to have the right questions. We enjoyed very much to spend time with our three children during our holidays in our country house in Faverdines since 1966 (3 hours from Paris), in the Alps since 1980 (Val Thorens), on the sea side in Rayol Canadel or visiting different countries (Germany, Portugal, Marocco, US) (Figure 3p. It was a fascinating time for us to see them growing so fast. They get very rapidly an autonomy which helped them for their future. All three children obtained very good jobs. Laurent, after the "Ecole des Arts et Métiers" (ENSAM), is an engineer at the CEA (Commissariat à l'énergie atomique) and is building the ITER facility in Cadarache. Anne and Céline earned very good diplomas from the school "Ecole Polytechnique Féminine" (EPF) and entered in the bank research and development department. In industry they have to work very hard until $8 \mathrm{pm}$. They sometime regret not having chosen a career in the research where you have a lot of work to do but you are your own manager. They saw their mother always working but they realized only later the freedom that the researchers have. By this time I was also involved in discussions about the ethic of research in a working group at the "Assemblée Nationale" (French Parliament) managed by Alain Lamassoure, an elected representative of parliament.

Now I have eight grand children and am happy to invite them to our different holiday resorts or to visit different countries where meetings take place (Figure 4). They particularly appreciate watching the solar eclipses and like very much the COSPAR General Assemblies with their space exhibitions. They discover that the English language is important to communicate with others. 


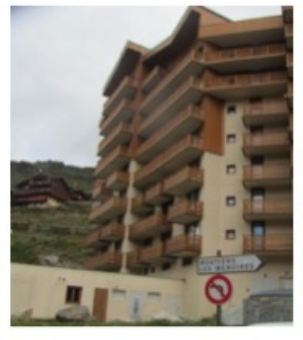

Val Thorens
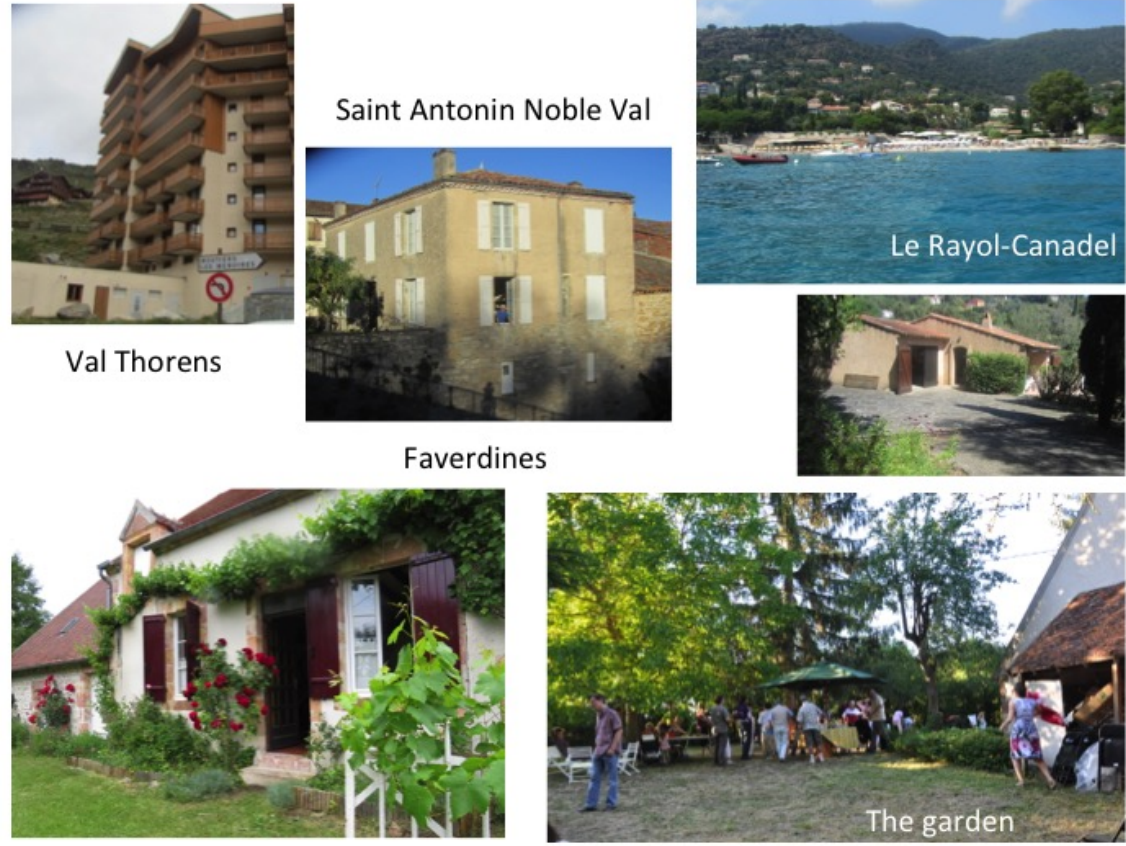

Figure 3. Where I spend my holidays with my children and friends and now where I am writing my papers and reviews: Val Thorens in the Alps, Saint Antonin Noble Val in Tarn et Garonne department, Le Rayol Canadel with its famous beach on the riviera, Faverdines a charming small house with a large garden that many of my colleagues know.

\section{Research}

\subsection{My concept of research}

In 1980 the Solar Maximum Mission (SMM) was launched. The researcher Einar Tandberg-Hanssen, a Norwegian who had emigrated to the US, and very fond of France, visited our laboratory (see his memoir - Tandberg-Hanssen, 2011). He was the principal investigator of the UVSP spectrometer onboard SMM. He demonstrated to us the interest to combine multi-wavelength observations to understand the activity of the Sun. These two events changed completely my research topics and my life. During the preceding ten years I have been mostly alone in my office, not traveling, and then I discovered foreigners working together and discussing together.

It was a new world for me, more appropriate to my life concept. E.Tandberg-Hanssen has been the main actor to open to me the NASA laboratories in US (GSFC, MSFC) and also to make strong contacts in Norway. Later on, I was the advisor of Jun Elin Wiik, a Norwegian student (thesis defense in 1993). And finally, because of the success of Jun Elin Wiik, I got a part time professorship in Norway from 1996 to 2006 working on filaments with Oddbjorn Engvold and his staff, e.g., Jun Elin Wiik and Yong Lin (PhD student). I was the second woman to teach physics at the University of Oslo in Blindern. I appreciated very much all my stays in Oslo between the fjords and the mountains with my colleagues: Oddbjorn Engvold, Olav Kjeldseth-Moe and Mats Carlson who became a "chevalier du test vin" in Burgundy due my sponsorship. 

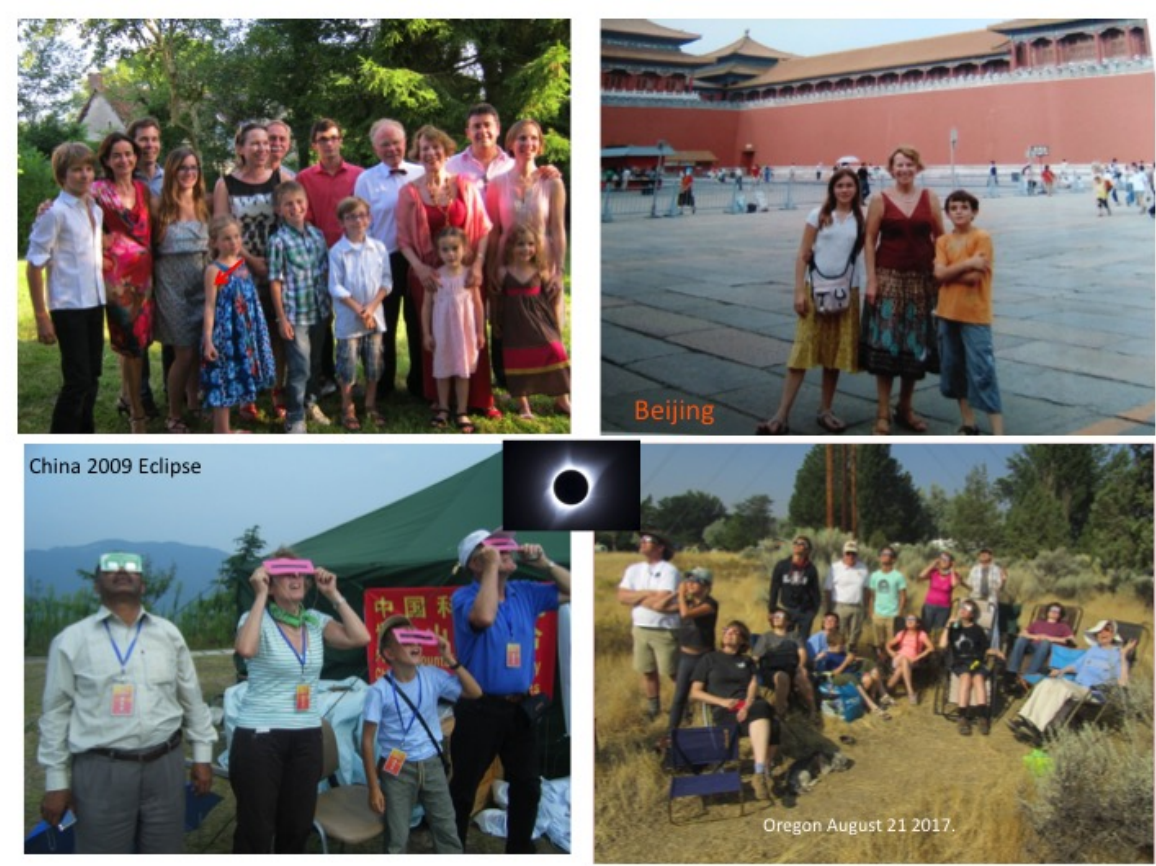

Figure 4. Top left, my family: me, my husband Eric with our 3 children, the spouse of our son and husbands of our two daughters and our 8 grand children in the garden at Faverdines, top right: visit of the Forbidden City with my grand children Camille and Adrien during COSPAR in 2006, bottom left, the eclipse in 2009 in Hangshou (China) with Wahab Uddin, me, Grégoire and my husband, bottom right: the eclipse on August 21 in 2017 in Madras (Oregon, US) with Sarah Gibson and Mark Miesh and their family seated on chairs, behind them, standing up, my family: Capucine, Grégoire, Eric, Gautier and me (in red tee shirt).

All my research was oriented according to the collaborations that I managed through bilateral research projects which brought me to visit many countries (US with Einar Tandberg-Hanssen, Art Poland, Dave Rust, and Leon Golub, Japan with Hiroki Kurokawal and Kazunari Shibata, Czech Republic with Petr Heinzel, Greece with Kostas Alissandrakis, Georgia Tsiropoula and Kostas Tziotziou, China with Fang Cheng, Argentina with Marcos Machado and later with Cristina Mandrini, India with Venkatakrischnan and now with Ramesh Chandra in ARIES, and recently in Korea with Tetsuya Magara at Kyung Hee University and Young Deuk Park in KASI. All these researchers brought and still bring to me a lot of knowledge in different aspects of astrophysics.

I visited China for the first time in 1991, I was invited by Xiaoma Gu for one month in Kunming and again in 2004 (Figure 6 top left panel). There I also met Jun Lin as a young student. In China at that time there was no xerox machine to copy the rolling paper where flare spectra of interest were recorded. I traced the spectra on a paper and in Meudon I digitized them by using a machine overlying each curve with a stiletto and published consequently a paper on post-flare loops (Gu et al., 1992). Before returning to France, I visited Beijing with Ai Guo Xian and Fu Qi Jun and Nanjing with Cheng Fang (see his memoir - Fang, 2018) who could speak French fluently after his stay in France in 1987. I have a nice story about the arrival of Cheng Fang in our laboratory. Jean-Claude Hénoux, our director at that time, received a letter asking him if we would agree to have a Chinese visitor for 2 years. We said yes. After three 

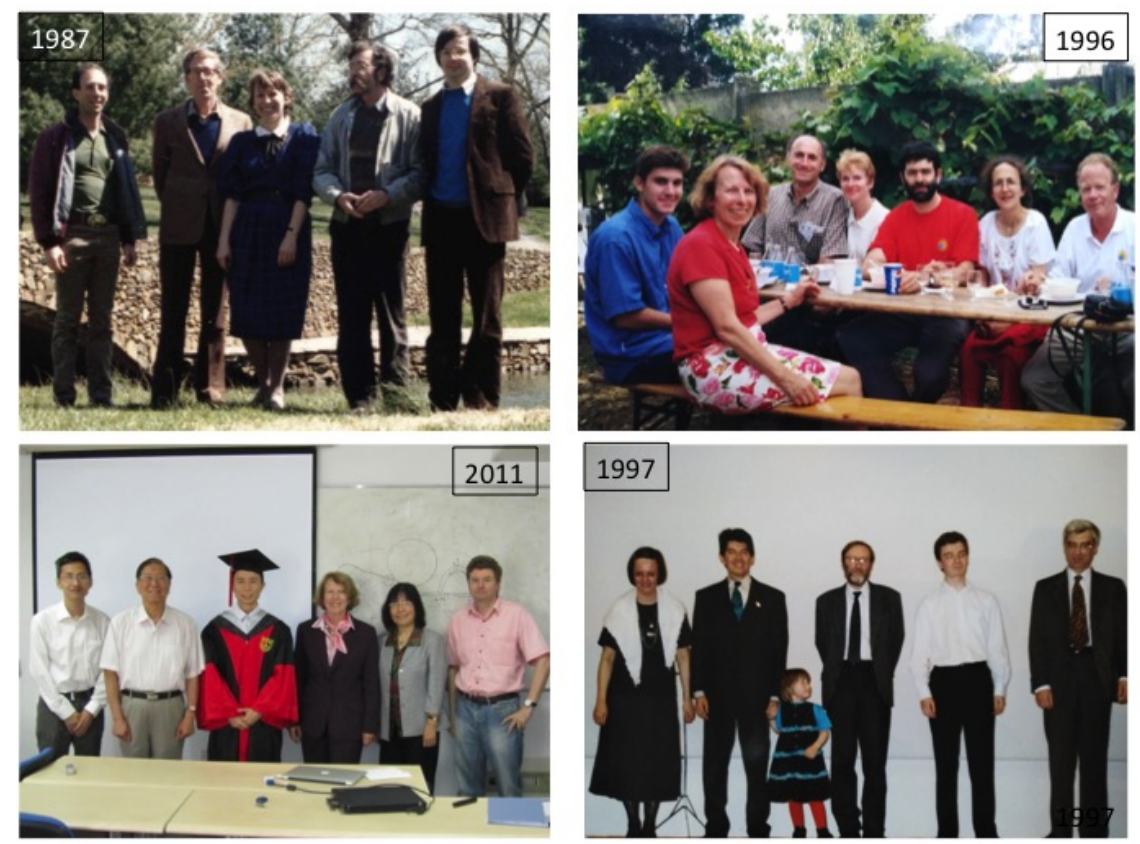

Figure 5. Top left, photo taken at the SMM Workshop in Airlie in 1987: Art Poland, Einar Tandberg-Hanssen, Brigitte Schmieder (BS), Guy Simon, Jean-Marie Malherbe (PhD student), top right, waiting for the eclipse in 1999 at Szombathely in Hungary: Spiro Antiochos with his son and wife, BS, Pascal Démoulin with his wife, Eric Schmieder, bottom left, Guo Yang defended his thesis in Nanjing 2011 with the jury: Mingde Ding, Cheng Fang, BS, Hubert Baty, bottom right, during the Aussois meeting in 1997, a concert was given by Véronique Bommier, Bernard Foing with his daughter, Petr Heinzel, Jean-Francois Mein and Pierre Mein.

months he received the same letter. He replied: Yes of course. In fact one was Cheng Fang and the other one was Feng Cheng from Kunming. Finally both came for two years. Later I visited China again, mainly for giving lectures to young students (Figure 6 right panels), and to attend meetings, e.g, the French-Chinese meetings in Xian and Shanghai, the 36th COSPAR General Assembly in Beijing, the first Chinese-European meeting in Kunming in 2017. I was co-advisor of two Chinese PhD students: Yang Guo (Figure 5 bottom left panel) and Jie Zhao who defended their theses in 2011 and 2014, respectively.

I should also mention my numerous trips to India. I was first invited in Nainital in 2005 by Wahab Uddin where I met Ramesh Chandra and Navin Joshi who are very good observers. I got an official collaboration (CEFIPRA) with Nainital, Udaipur, and Oooty. This allowed me to work and have many exchanges with colleagues and their students in India. I was invited to watch the eclipse in 2009 with Grégoire, my grandson by W. Uddin and Siraj Hasan in Hangshou where we arrived after a long trip during the night. They were still waiting for us. Siraj said, Brigitte did not cancel her trip, so we have to wait for her all the night. And we were rewarded with clear skies (Figure 4 top bottom panel). I will refer later to our scientific results.

In addition, we had individual foreign visitors who also interacted strongly with our team and our PhD students. I will refer to them later to this memoir. All my PhD students (Jean-Marie Malherbe, Pascal Démoulin, Guillaume Aulanier, and Etienne 

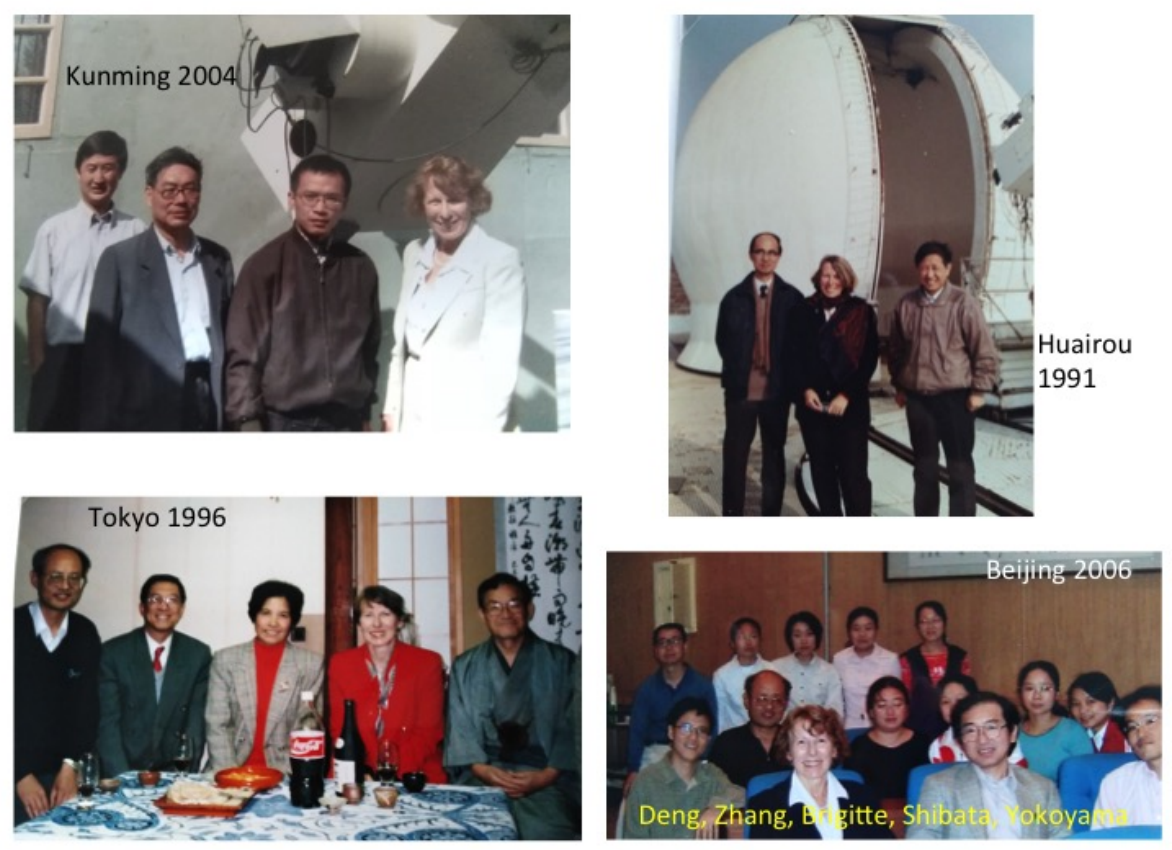

Figure 6. Top left, Kunming in 2004: Kejun, Li, Xiaoma Gu, Xiaoyu Zhang and Brigitte Schmieder (BS), top right, Huairou 1991: Fu Qi Jun, BS, Ai Guo Xian, bottom left: dinner by Eijero Hiei (on the right) in Tokyo in 1993: Hong Qi Zhang, Cheng Fang, Tang, BS, bottom right: after the 36th COSPAR 2006, during lectures in Beijing: first row, Yuanyong Deng, BS, Shibata, Yokoyama, second row: Hong Qi Zhang, Guiping Ruan, third row: Xinming Bao and students.

Pariat) could work with them. That was an excellent training for my students and we maintained good relationships and even friendships with all of the foreign visitors. Our French team developed as an extended group with all these researchers without frontiers. With this very friendly and active ambience, and due to their excellent skills, all my French PhD students cited above got a permanent job at the Paris Observatory and formed the roots and core of the present solar group.

\subsection{Time of SMM and J.M.Malherbe -1980's}

Jean Claude Pecker encouraged Guy Simon to submit a proposal to the Guest Investigator program committee of the Ultra Violet Spectrometer (UVSP) on UV waves after flares. The proposal was successful and we got a few weeks of observing time during several years. Guy Simon and I went to the building 7 or 21 at NASA/GSFC in Greenbelt near Washington DC for planning our observations (Figure 5 top left). Each morning we had a meeting to decide the target of the next day to determine the pointing of the telescope. The members of the committee hated our proposal which required to move away from the flare site but E. Tandberg-Hanssen regularly reminded them that they had accepted the proposal so it should run. On Sundays Guy Simon was not allowed to enter the NASA compound because of his involvement in politics and I had to discuss alone the target of the day. These observations were coordinated with Meudon using the MSDP at the solar tower. Christian Coutard and Roland Hellier 


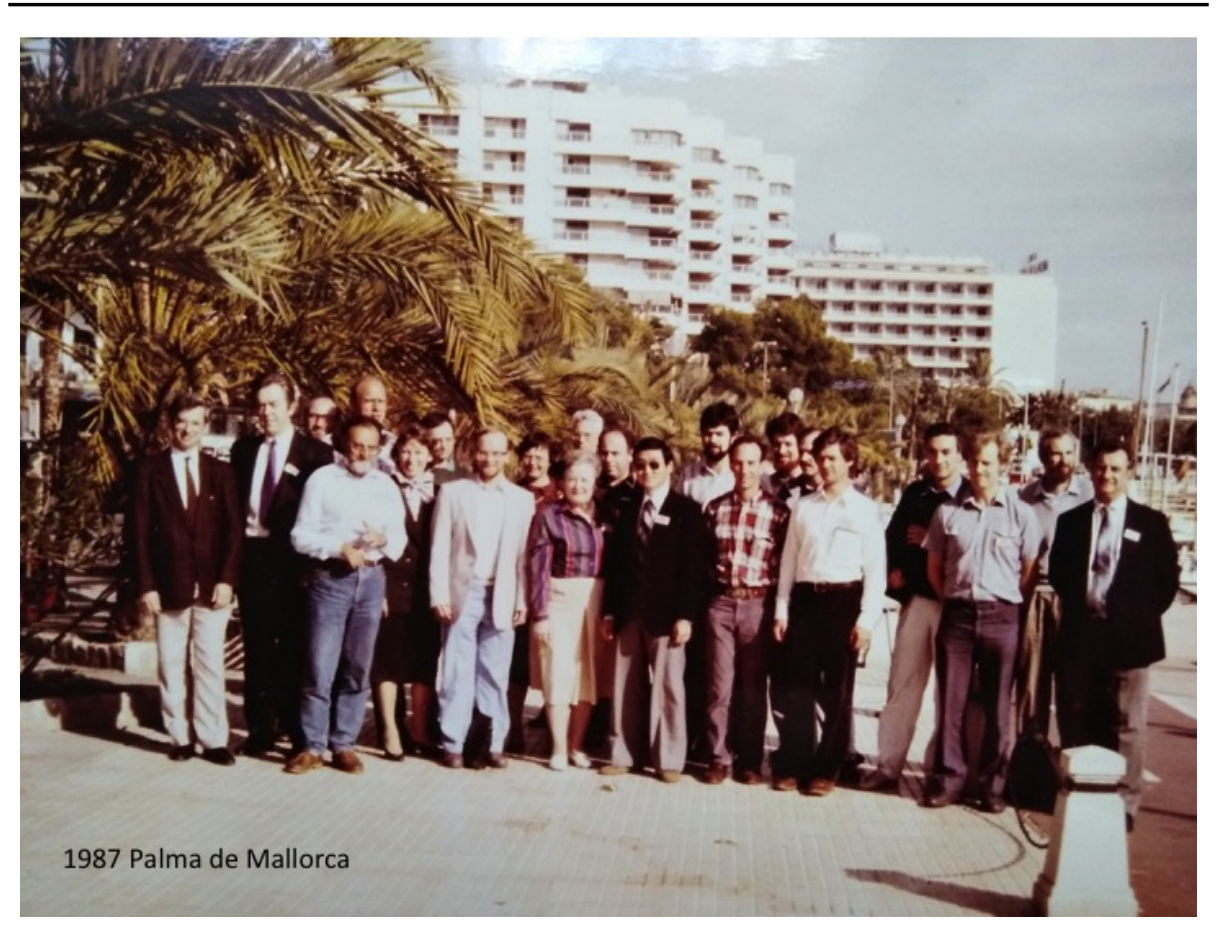

Figure 7. Workshop on the Dynamics and structure of solar Prominences in Palma de Mallorca in November 1987, first row, from left to right: Pierre Mein, Oddbjorn Engvold, Ulrich Anzer, Brigitte Schmieder, Eric Priest, Marie José Martres, Guillermo Vizoso Miguel del Sola, Art Poland, Horst Balthazar, Alan Hood, Bogdan Rompolt, second row, José Ballester, Eberhard Wiehr,Jean Marie Malherbe, Véronique Bommier, Jean Louis Leroy, -, Pascal Démoulin, -, -, Fernando Moreno Insertis, Pierre Gouttebroze.

were our observers and they had to develop wrapping black and white films in dark rooms every day. Besides it was not easy for Pierre and Nicole Mein to receive the coordinates of the target. In the 1980's Internet communications were just starting. In Meudon there was only one telephone-modem in the computer center with which we could communicate with the operation center at NASA/GSFC.

The aim of the proposal was to study the propagation of waves in UV after flares. It was completely an unknown topic at that time, only the Moreton waves were observed. We were really pioneers on this topic. To achieve our goal we requested that after the flare flag we moved the center of the field of view to the edge of the camera and wait for the passage of the wave. With a Fourier transform code we tried to detect the waves. After nights and nights spent on the computer no wave was detected, due to the low cadence of the instrument. However the data that we obtained were generally centered on filaments so we started to work on filaments. I met many scientists during that period with whom we continued to work later, e.g, George Simnett, Art Poland (Figure 5 top left). All became good friends and visited France frequently. Recently I started to work again on these famous EUV waves that have been discovered with the next satellite instruments in UV onboard SOHO /EIT and SDO/AIA (Delannée et al., 2014, Chandra et al., 2018).

During all these years (1980's) I was organizing coordinated campaigns observing simultaneously on the ground with the MSDP either in Meudon or at Pic du Midi with Pierre and Nicole Mein. With the MSDP we were observing mainly in the $\mathrm{H} \alpha$ 
line jointly with the UVSP instruments in the EUV lines like C IV for Dopplershifts of the plasma at $10^{4}$ to $10^{5}$ degrees. Different topics were open to us: the understanding of the dynamics of the cool plasma in prominences, cool jets or surges, and flares. We reported our results in many conferences, e.g, Airlie (US) in 1987 (Figure 5 top left), in Palma de Majorca in 1987 (Figure 7), in Aussois in 1997 (Figure 5 bottom right), in Hungary for the eclipse in 1999 (Figure 5 top right).

Jean-Marie Malherbe came in the group to start a thesis in the early 1980's. He was interested in instrumentation, physics, and computations. He worked with us on the dynamics of prominence plasma condensations and on the dynamics of flare ribbons. We had a great chance to meet two bright theoreticians who visited us many times, Mike Raadu and Terry Forbes. They brought innovative ideas during fruitful discussions. Our measurements from the MSDP were quantitative and not only descriptive, therefore they were interested to check their ideas or computations. A very intensive collaboration with theoreticians started at that time. It was the seed for the birth of the Meudon solar MHD group in the future. With the observations at Pic du Midi combined with the C IV data of the UVSP provided by Einar Tandberg-Hanssen and Art Poland we could measure the dynamics in fine structures of filaments, up and down motions in the feet and horizontal flows along the axis of the filament, rotation along the filament axis in case of disturbances, and oscillations in filaments (Martres et al., 1981; Malherbe, Schmieder, and Mein, 1981, Malherbe et al., 1982 Mein et al., 1982; Malherbe et al., 1983 Schmieder et al., 1984 Schmieder et al., 1985 Simon et al., 1986 Malherbe et al., 1987). Mike Raadu proposed to us some simple models to explain the drainage of cool matter when the loops passed through the photosphere, the empty basket model (Raadu et al., 1987a), and also a destabilization model of a filament showing twisted motions in a flux rope which lifted up as in the torus instability advanced later on by Tibor Török (see Török et al., 2011 and references therein). The cause of the filament destabilization could be explained by photospheric motions (Raadu et al., 1987b Raadu et al., 1988).

With George Simnett and Einar Tandberg-Hanssen we understood that surges formed of cool plasma observed in $\mathrm{H} \alpha$, and jets of hot plasma visible in UV and Xrays, were co-aligned and had similar velocities (Schmieder et al., 1982; Schmieder et al., 1983: Schmieder et al., 1984 Schmieder et al., 1988a; Schmieder et al., 1988b Schmieder et al., 1993, Fontenla et al., 1994 Schmieder et al., 1996c). At the same time I worked also on surges with American scientists who were visiting us: Leon Golub and Spiro Antiochos (Schmieder, Golub, and Antiochos, 1994). Both of them became good friends of the group and we could apply for post doc positions at NRL in Washington and visitorships at SAO at Harvard for our students for their training in MHD, e.g, Guillaume Aulanier, Etienne Pariat, and Sophie Masson.

Detection of transverse oscillations in filaments in $\mathrm{H} \alpha$ was achieved by Bill Thompson using MSDP observations (Thompson and Schmieder, 1991). The SMM mission launched in 1980 was lost during a few years before a cosmonaut going out of the Space Shuttle could recover it by using a Canadian robotic arm. It was an unbelievable rescue for the spacecraft. Our group (Nicole Mein, Pierre Mein, Guy Simon, Jean-Marie Malherbe, and I) could work with the data until 1989 with our friends of the UVSP spectrometer. The Meudon solar tower was closed in the 1990's and could re-open only in 2003 when THEMIS, the French-Italian magnetograph, on Tenerife, the Canary islands (opened in 1997) was fully operating and also after the films recording was replaced by J.M.Malherbe with a CCD camera, more convenient for the observations. J.M. Malherbe was a very fast thinking student and it is always interesting to work and discuss with him and Thierry Roudier in Toulouse the horizontal flows below filaments (Roudier et al., 2008, Schmieder et al., 2014b Roudier et al., 2018). 
Our work with Terry Forbes concerned mainly flares. He and Jean-Marie Malherbe developed an MHD model (using the code SHASTA) to explain reconnection in the corona and the cooling of the plasma inside the reconnected loops (Forbes and Malherbe, 1986). The observations of the flare ribbons that I had obtained in Meudon followed exactly their predictions. It was the discovery of the evaporation of the chromosphere with two phases: the impulsive phase and the gentle evaporation phase in post-flare loops (Schmieder et al., 1988c, Schmieder et al., 1990). I had the chance to meet again Terry Forbes in New Hampshire and during conferences, e.g, in Romania in conferences organized by Cristiana Dumitrache (Figure 8 bottom right panel). In the 2000's we could confirm the predictions of Forbes and Malherbe simulations concerning the chromospheric evaporation by using NLTE (non local thermodynamic equilibrium) radiative transfer computations and data of SOHO/CDS with Arek Berlicki and Guilio DelZanna (Berlicki et al., 2005, del Zanna et al., 2006). In the 1980's we were pioneers in this field of research.

Other interesting topics involved collaborations with our Greek colleagues. While working on sunspots the Evershed effect was found to reverse in the chromosphere compared to the direction observed in the photosphere (Alissandrakis et al., 1988 Dere, Schmieder, and Alissandrakis, 1990). With Georgia Tsiropoula we analyzed carefully the MSDP data concerning the chromospheric fine structure to understand if mottles on the disk were the same as spicules visible at the limb and had fruitful discussions with Petr Heinzel who was not always in agreement with our results (Tsiropoula, Alissandrakis, and Schmieder, 1993 Tsiropoula, Alissandrakis, and Schmieder, 1994 Tsiropoula and Schmieder, 1997. Heinzel, Schmieder, and Mein, 1992).

From 1990 to 1996, I was elected as vice president of the European Solar Physics Meeting (ESPM) organization. As ESPM vice president I organized two meetings with president George Simnett in Catania (1993), and in Thessaloniki (1996). In 1992 I was also elected as president of the Joint Organization of Solar Observations (JOSO) in Europe until 2002. JOSO had been created to develop collaborations between the solar observatories in Europe. Therefore I organized each year a meeting, e.g, Trieste in 1994 (Figure 9 top left and bottom right panels), Potsdam in 1999 (Figure 8 top right panel). Because the attendance increased to more than 150 participants, I wrote proposals to European Union to be sponsored. I get two successful EU contracts during my JOSO presidency to organize successively two series of European conferences: three of them on "Advances in Solar Physics" in Tenerife (1996), Preveza (1997), and Catania (1998) published in PASP journals and two of them on "Solar cycle and Space Weather" (SOLSPA) in Tenerife (2000) and in Vico Equenze near Napoli (2001) published by ESA publications.

The main objective of JOSO was to involve the solar physicists of all the European countries to create a data-base of solar observations, and to define the characteristics of a future large European telescope. Now this objective is pursued by the European Association for Solar Telescopes (EAST) consortium, which has been charged to build the European Solar Telescope (EST) (4m mirror). Today the plan is to start construction in 2021 and to achieve the first light in 2027. Let us see what will the future of EST be.

Between 1980 and 1992, I was the public outreach manager of the Observatoire of Paris. I was in charge of press releases and the organization of visits to the Observatoire de Paris in Meudon of students and the general public. I organized night shows for journalists, lectures for teachers on Wednesdays and also open doors for the public once a year. People appreciated these initiatives and it happened that we had more than one visit of 30 persons each day. Particularly memorable the open house in May 1985 when 10000 persons visited the observatory during the weekend (Figure 10). I 

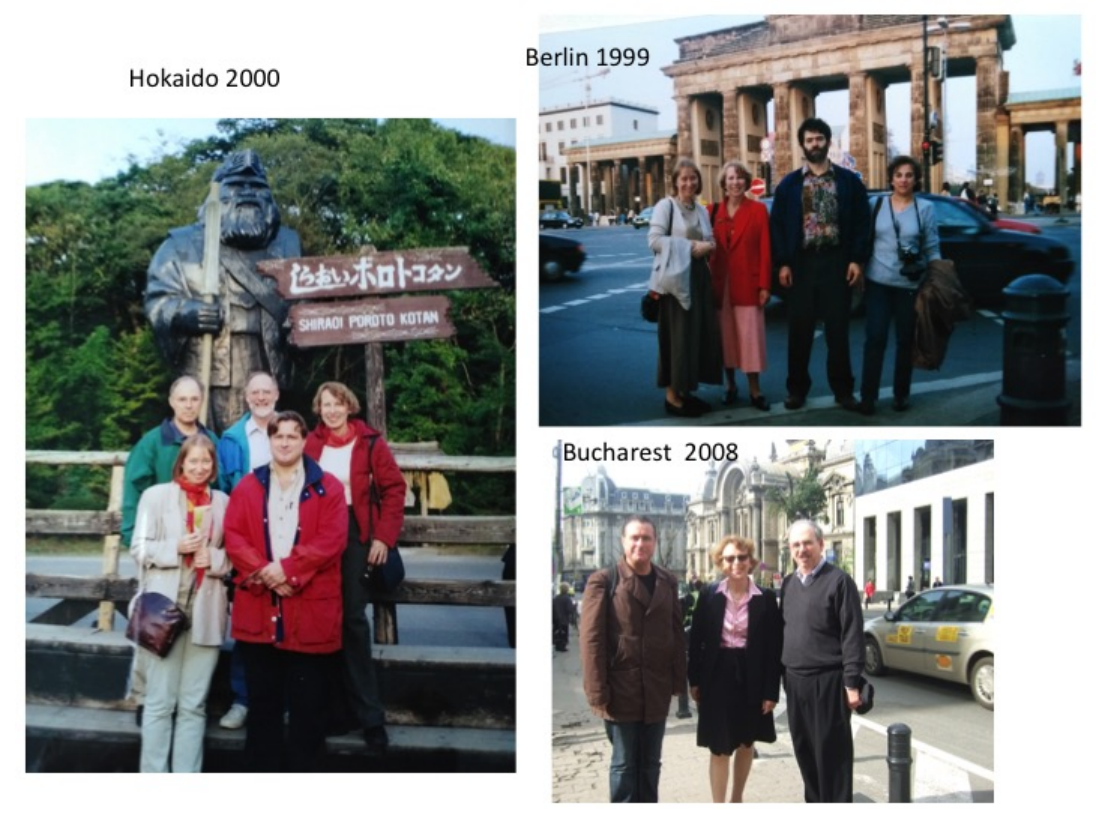

Figure 8. Left, Hokaido in Sapporo Island visit of the Inuits after S-Ramp meeting in 2000 organized by the SCOSTEP to summarize the results of the STEP program, first row, Lidia van Driel, Stefaan Poedts, second row, Ed Cliver, Dave Webb, Brigitte Schmieder, top right Berlin, during a JOSO meeting in Potsdam in 1999: Lidia van Driel, BS, Pascal Démoulin, Cristina Mandrini, bottom right, after the Space Weather meeting in Bucharest in 2008: Stefaan Poedts, BS, Terry Forbes.

got funding from the Ministry of the Culture for renovating the big dome (over the Meudon castle) and the refractor built by Jules Janssen.

But after the tornado in Paris in December 1999 the dome lost part of its coverage and despite some ministry funding, it is still under rehabilitation and no visits are possible anymore.

\subsection{Time of Yohkoh and P. Démoulin - 1990's}

My work all along my career was guided by the new solar missions launched to resolve the problem of coronal heating. It is still an up-to-date topic as we heard on TV on August 12, 2018 when the Parker Solar Probe was launched to approach the Sun, the main goals being to understand the heating of the corona, and to measure in situ the solar wind for future journeys of humans to Mars or the Moon.

After SMM, Yohkoh, the Japanese-American mission was launched in 1991 with several instruments. I was mainly involved with the Soft X-ray Telescope (SXT). During that time period TRACE (1998) was dedicated to observe the Sun in UV. Both SXT and TRACE observed partial fields-of-view and we needed to select the targets. I was guest investigator for both missions and organized multi-wavelength campaigns; Yohkoh was open to foreigners only after 1993. I went to the Institute of Space and Astronomical Science in Fuchinobe several times learning how to process the data. It was the beginning of the solar software (SSW).

I met many scientists again: Bob Bentley (Figure 9 bottom right), Jim Lemen, Lidia van Driel (Figure 8 left) and by chance a few Japanese in Mitaka: Tadashi Hirayama, 

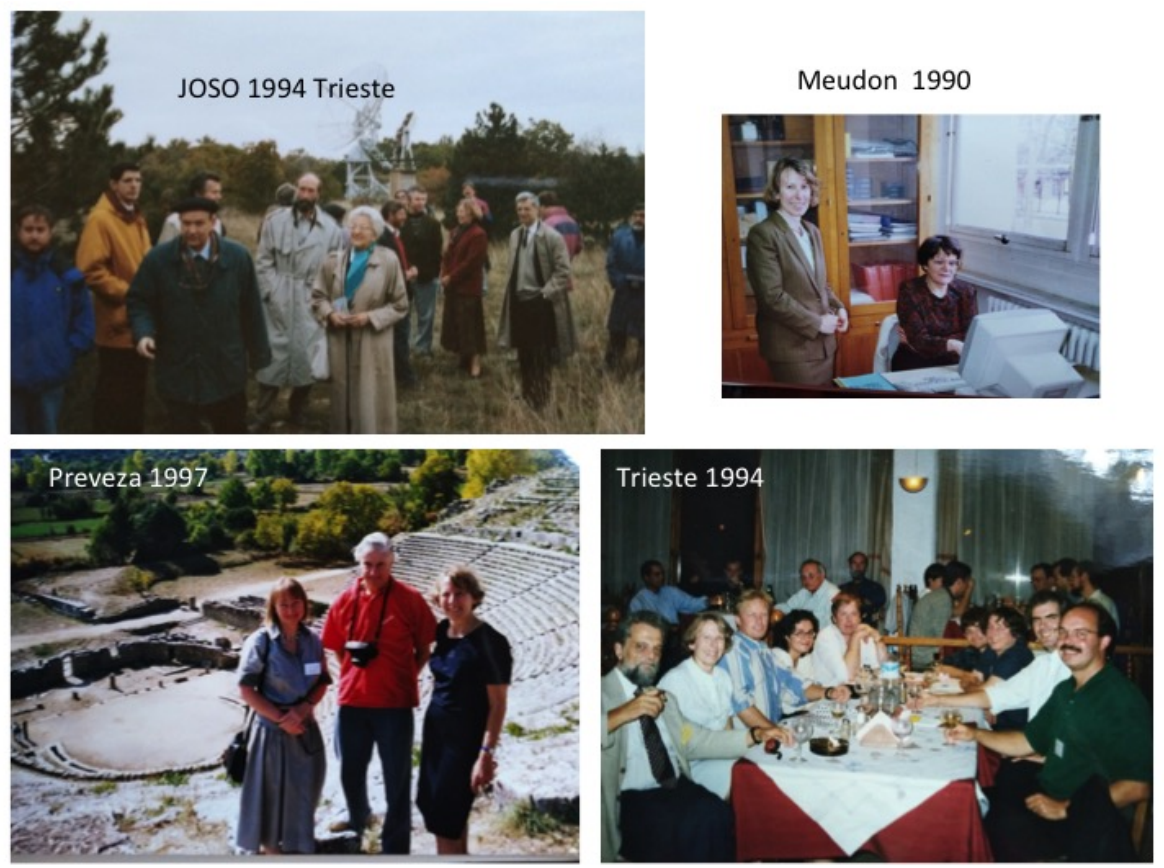

Figure 9. JOSO group visiting the radio telescope in Trieste in October 1994: from left to right we can recognize some scientists: Bojan Vršnak, Mauro Messerotti, Paolo Zlobec and behind Axel Hofmann, Peter Brandt, Edith Muller -former president of JOSO-, behind, Richard Muller, Thierry Roudier and BS (president of JOSO), Janusz Sylwester, Pierre Mein, Lucas Vlahos, top right: BS and Nicole Mein working in Meudon observatory, bottom right, dinner during a JOSO meeting in Trieste in 1994: Costas Alissandrakis, BS, Paul Brekke, Greek scientist, Bob Bentley, -, Philippa Browning, David Alexander, Bernard Fleck, behind Meir Semel and Peter Brandt, bottom left: Lidia van Driel, George Simnett, BS during the Preveza Euroconference in 1997 on Advances in solar Physics visiting Dodone.

Eijero Hiei (Figure 6 bottom left), Takashi Sakurai, Hiroki Kurokawa and Kazunari Shibata (Figure 11) (see the paper on surges by Schmieder et al., 1995b) and those on X-ray bright points (van Driel-Gesztelyi et al. Mandrini et al. | 1996, 1996). The main topic that we worked on was flare-loop formation. For the coordinated campaign with Yohkoh we (myself, J.M. Malherbe, and P. Mein) were observing with the MSDP at the "spectro tourelle" at the Pic du Midi in the 1990's. We obtained very fine observations of flare loops with the MSDP in June 26, 1992 to compare with the Yohkoh loops. These observations led to more than six papers with Jun Elin Wiik, Jean Marie Malherbe, Lidia van Driel, and Petr Heinzel (Schmieder et al., 1995a: Schmieder et al., 1996b: Wiik et al., 1996, Wiik et al., 1997. Malherbe et al., 1997| van Driel-Gesztelyi et al., 1997). I had already started with Petr Heinzel to work on plasma conditions in post flare loops (Heinzel, Schmieder, and Mein, 1992). We continued and computed the theoretical times to cool the X-ray loops to $\mathrm{H} \alpha$ temperature by radiative cooling and conduction (Schmieder et al., 1995a) and could confirm what the model of Terry Forbes predicted, that the hot loops visible after reconnection in X-rays with Yohkoh were cooled down to $10^{4} \mathrm{~K}$ after a certain time and observed in $\mathrm{H} \alpha$. Other papers appeared later on post flare loops visible after the impulsive phase of flares (Gu et al., 1997, Schmieder, Fang, and Harra-Murnion, 1998). 


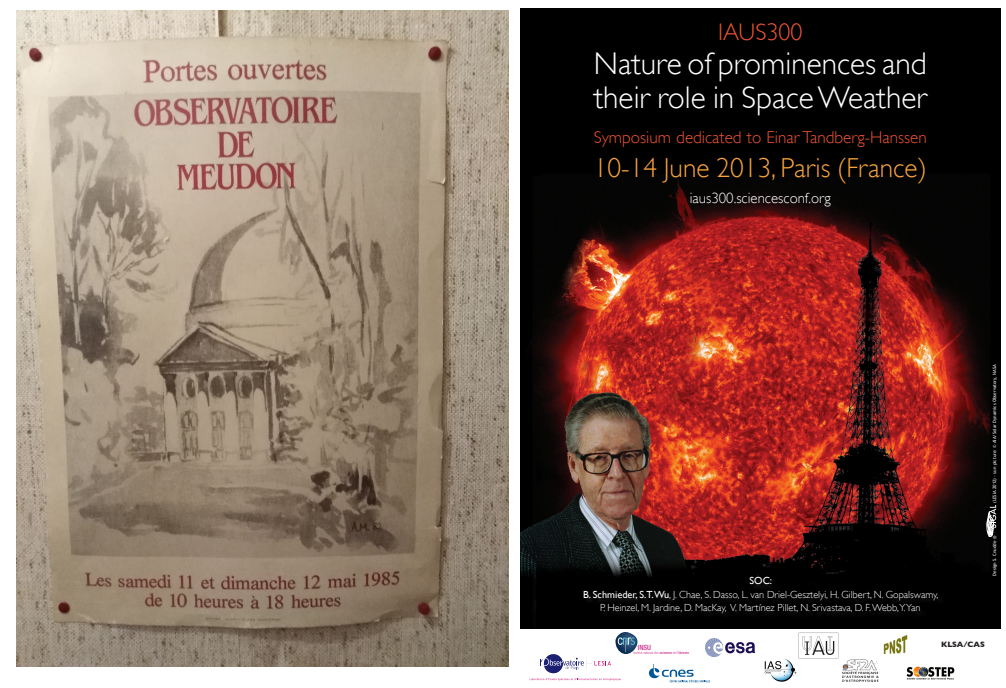

Figure 10. Left, poster for the open house on 11 and 12 May 1985 in Meudon (drawn by André Mangeney), right, poster for the IAU S300 symposium in Paris dedicated to Einar Tandberg-Hanssen (drawn by Sylvain Cnude).

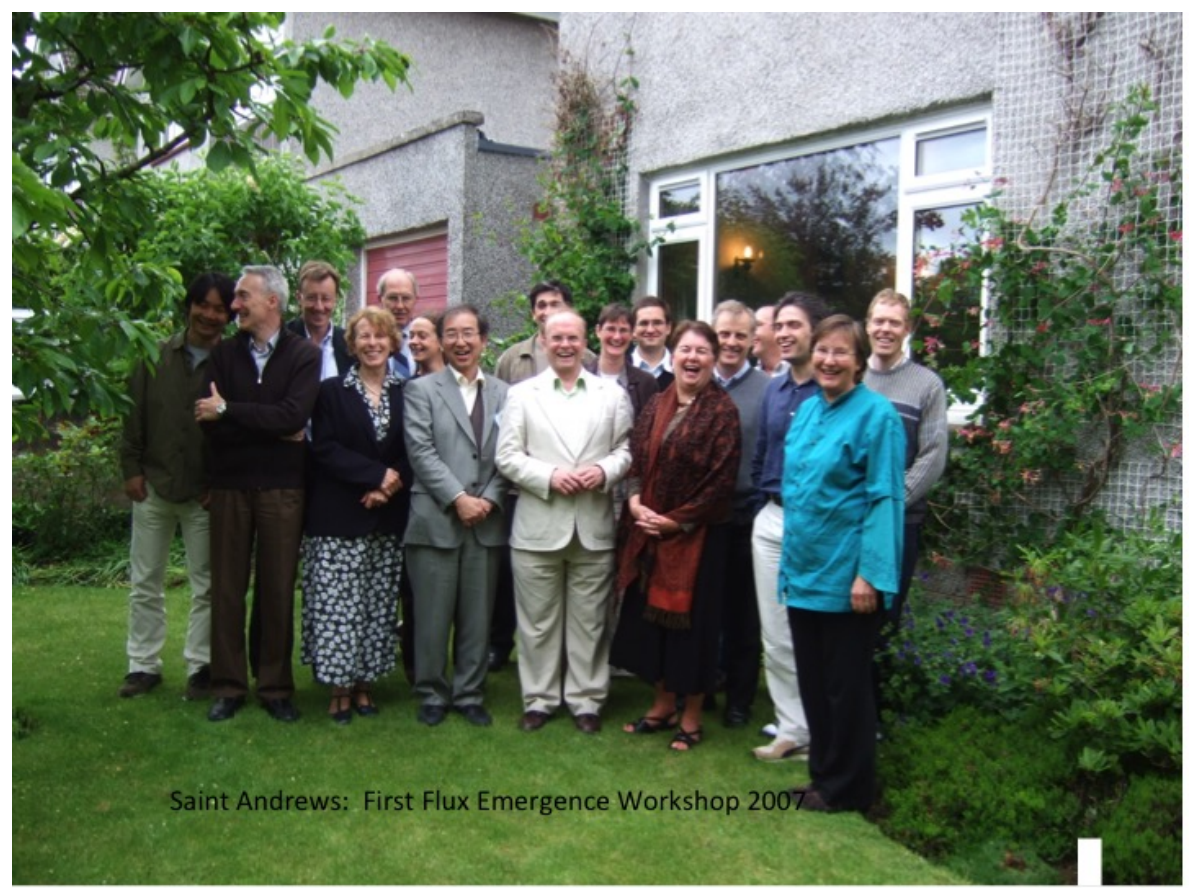

Figure 11. St. Andrews 2007, first Flux Emergence Workshop, in front of the house of Eric Priest, from left to right, first row: Isobe Hiroaki, Fernando Moreno Insertis, BS, Shibata Kazanari, Eric Priest, Gael the wife of Dave Rust, Clare the wife of Eric, second row: David Hughes, Dave Rust, Laurène Jouve, Evy Kersale, Clare Parnel, Etienne Pariat, Alan Hood, Dana Longcope, Sacha Brun, Klaus Galsgaard. 
However during the loss of SMM and before the launch of SOHO (1996), the space data were not easy to access and it was the period when Jean Heyvaerts, who was the responsible professor assigning the $\mathrm{PhD}$ students in different laboratories proposed to me to be an advisor of PhD students. Heyvaerts appreciated the way that I was training students on solar observations with an open view. In the first year, I got a very bright Chinese student Z.S. She (She, Malherbe, and Raadu, 1986) but he wanted to work only on turbulence so after one year he moved to Nice with Uriel Frish. The next year I got Pascal Démoulin and Heyvaerts told me that I should be able to teach him to explain all the "zoo of solar physics: flares, sunspots, plages, surges and eruptions". It is with Pascal that our solar MHD group was born finally. For his PhD I had to find a theoretical group which could give to him a proper theoretical background. I had good relationship with theoreticians in Florence, e.g., Georgio Einaudi, Franca and Claudio Chuideri. Pascal was very enthusiastic to interact with them. However after one year no paper has emerged. My famous question "Where is the paper" was asked. At that point he had only one but very impressive paper with me and Mike Raadu on the role of parallel and perpendicular conduction in the stability of filament fine structures (Démoulin et al., 1987). Because I knew that he had to publish if he wanted a permanent position, I asked Eric Priest if Pascal could visit him in St. Andrews. Eric Priest reported in his memoirs: "It was a real pleasure to work with him, since he is so bright and couples a superb physical understanding with great technical skills" (Priest, 2014). And this is how and why a solar MHD group developed in Meudon. Pascal and myself were invited to many meetings, e.g., in Potsdam for the JOSO meeting in 1999 (Figure 8 top right panel) and in Hungary for the 2009 eclipse (Figures 5 top right panel).

As a PhD student, P. Démoulin began to work with the general topic of prominence equilibria, the formation of dips and their support and loss of equilibrium or instability in a force-free field (Démoulin and Priest, 1988: Démoulin, Malherbe, and Priest, 1989 Démoulin and Raadu, 1992 Démoulin and Priest, 1993). These ideas were further developed by Guillaume Aulanier in his thesis (Aulanier and Démoulin, 1998. Aulanier et al., 1998a: Aulanier et al., 1999, Aulanier and Schmieder, 2002). And so later on Pascal Démoulin kept contributing intensively to the theoretical training of my other PhD students. By that time (1990's) P. Démoulin was also interested in the topology of the flaring active regions and pioneered research on reconnection without null points, along the quasi-separatrix layers (QSL) (Démoulin et al., 1996, Démoulin et al., 1997). This theory was applied to many observations, by the successive scientists of our group, e.g., Guillaume Aulanier, Etienne Pariat, Kevin Dalmasse, and also in Argentina, China and India (Deng et al., 1999; Mandrini et al., 1996; Schmieder et al., 1997; Moore et al., 1997; Mandrini et al., 1997|| Berlicki et al., 2004; Mandrini et al., 2006 |Schmieder et al., 2007b). This work continues to be developed by the Argentinian group, still with linear force-free field extrapolations. By comparing the similarity of the locations of QSLs for a few case-studies using linear and non-linear force-free field approach, the robustness of the QSLs, regions of intense electric currents before flares has been demonstrated (Mandrini et al., 2014, Chandra et al., 2011, Dalmasse et al., 2015 Joshi et al., 2019).

In the 2000's P. Démoulin applied the theory of the conservation of magnetic helicity to active regions and showed that the excess of magnetic helicity in twisted flux ropes can be expelled by coronal mass ejections (CME). This created a stream of various papers in our group (see the Pariat papers in his thesis, Chandra et al., 2009, Zhao et al. 2014) and more recently papers by Kevin Dalmasse (Dalmasse et al., 2018). It is still a hot topic in the group with our new $\mathrm{PhD}$ student Luis Linan.. 

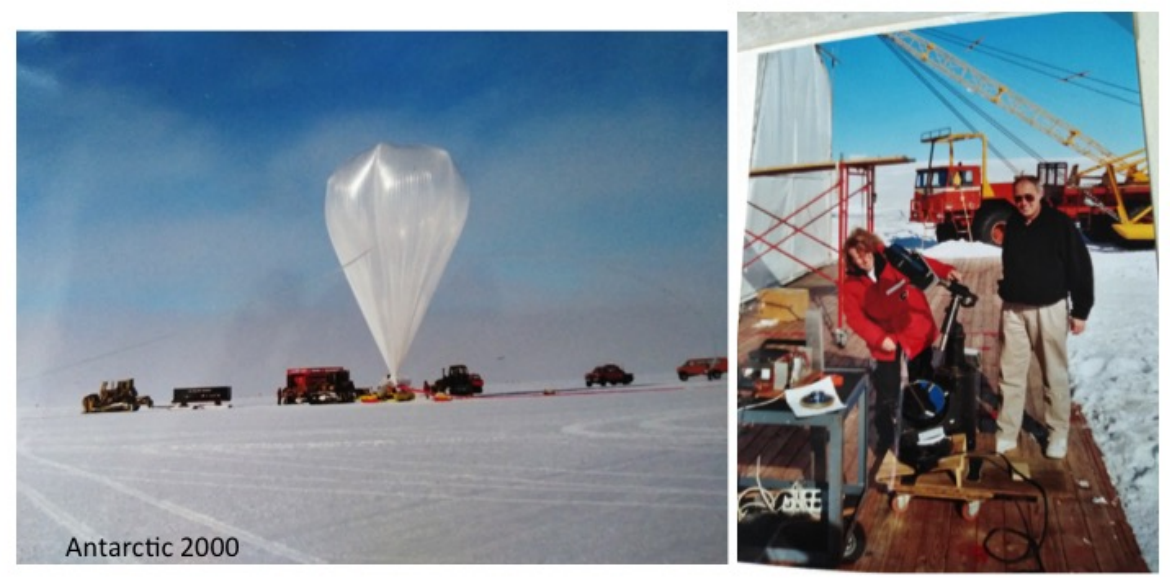

Figure 12. Left, launch of the Flare Genesis Experiment hung below a balloon in MacMurdo (Antarctica ) in 2000, right: Brigitte Schmieder and Dave Rust adjusting the coelostat in the barn before the launch.

\subsection{Flux emergence - Flare Genesis Experiment- E. Pariat - 2000's}

The Flare Genesis Experiment (FGE) was a balloon-borne telescope that made a journey of 17 days around the South Pole at $36 \mathrm{~km}$ altitude in the sky (Figure 12). This experiment was a precursor of the recent SUNRISE instrument. FGE was a Fabry Perrot instrument with which we could observe the chromosphere in the $\mathrm{H} \alpha$ line and a magnetograph which registered the Stokes parameters. Dave Rust was the PI of the different flights of this experiment. In 1998 the balloon came down in the French territory "Terre Adélie" in Antarctica just a few days before the closing of the base. Dave Rust did not have the official contacts between NASA and the CNES needed for the telescope to be be retrieved. Finally he phoned me on a Sunday asking me if I could help. I called Roger Gendrin in Brest and everything went smoothly. The observations were recovered and send back to MacMurdo by using a small French airplane. In 2000 Dave proposed to me to participate in the second flight of FGE in case similar problems with the French territory arose. I spent a month at MacMurdo base helping to adjust the instruments, and, after the launch, to define the targets for FGE, jointly with observations obtained with TRACE and Yohkoh. Dave Rust was a fantastic manager and organized our stay (only six persons at the base) very smoothly. I thank him very much to give me the opportunity to go Antarctica.

We were lucky to observe newly emerging magnetic flux during this flight. With my new PhD student Etienne Pariat, we could, for the first time, derive the undulatory behavior of the flux tubes as they emerged through the photosphere, by analyzing the magnetic vectors and constructing the magnetic field in the corona using linear forcefree extrapolation. We also located the heating points where magnetic reconnection 

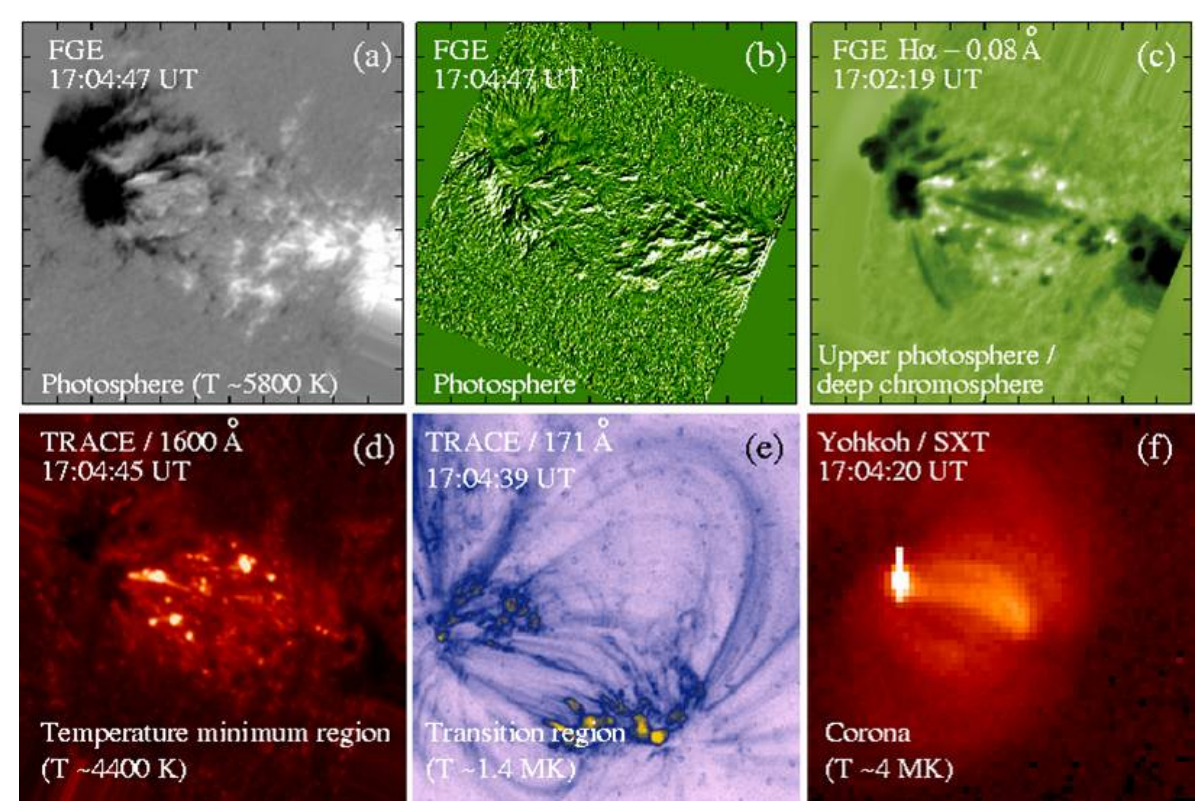

Figure 13. Flare Genesis Experiment results: (a) magnetic field in the photosphere, (b) electric currents in the photosphere, (c) Ellerman bombs in $\mathrm{H} \alpha$ and joint observations obtained with (d) TRACE intensity of the transition region (e) Yohoh/SXT in the corona, (f) and SXT observations of very hot loops in the corona (Pariat et al 2004,Schmieder et al 2004).

occurs and the cooling and heating loops at different temperatures over the emerging flux region (Georgoulis et al., 2002, Pariat et al., 2004, Schmieder et al., 2004a) (Figure 13). Now with SDO/AIA and HMI it is possible to see all the UV bursts over the full disk produced by flux emergence. My more recent PhD students, e.g., Zhao Jie and Michalina Grubecka continued this work as a part of their theses to determine where the reconnection really occurs, in the photosphere or higher up, based on nonlinear force-free extrapolation as well as with the NLTE modelling of Ellerman bombs (EBs) provided by Arek Berlicki and Petr Heinzel (Pariat et al., 2007. Zhao et al., 2017, Grubecka et al., 2016).

We summarized the main results of these works in a review on emerging flux (Schmieder, Archontis, and Pariat, 2014) and a review on UV bursts (Young et al., 2018). The first workshop on flux emergence (FEW) has been organized in St. Andrews to celebrate the success of Flare Genesis Experiment around Dave Rust in 2007 (Figure 11) and since that time a FEW is organized every two or 3 years. In 2019 it held in Japan without Dave who passed away during the winter of 2019.

\subsection{Prominence studies - Jun Elin Wiik - Petr Heinzel - from 1990 to now}

In the early 1990's Einar Tandberg-Hanssen asked me to be the advisor for the thesis of Jun Elin Wiik, a Norwegian student of Eberhart Jensen in Oslo. He recommended that we worked on the fascinating structures called prominences which have a long history since their discovery by Secchi (1875-1877 Le Soleil) and the successive classifications existing on material protruding over the limb (Tandberg-Hanssen, 1995). Jun Elin started to work on the characteristics of prominence plasma by deriving the electron 
density (Wiik, Heinzel, and Schmieder, 1992). Then she studied the dynamics in filaments using observations made with the MSDP at the "spectro tourelle" at Pic du Midi. We discovered flows in both directions along filament threads, that would be called "counter streaming" flows seven years later (Schmieder, Raadu, and Wiik, 1991). Using the UV data of the rocket-launched High Resolution Telescope Spectrometer (HRTS), she computed the differential emission measure in prominences and uncovered their multiple sub-resolution thread morphology (15 to 30 threads per pixel) (Wiik, Dere, and Schmieder, 1993). This former result has been, more than ten years later, the basis of the development of multi-thread models (Gunár et al., 2007).

After her thesis defense in 1993 Jun Elin Wiik got a post-doc in Norway and I obtained a part time professor fellowship in Oslo in 1996. It was the time when SOHO was just launched. SOHO is a fantastic mission; its coronagraph LASCO is still working in 2018. However I was more interested in the spectroscopy data obtained with SUMER. I spent a lot of time during my stays in Norway processing the data by using the software developed by the Norwegian group, e.g., Mats Carlson, Oddbjorn Engvold, and Olav Kjeldseth-Moe. The SUMER data were obtained during coordinated campaigns focused on filaments and prominences with the Swedish solar telescope (SST) at La Palma, the MSDP at Pic du Midi, and the space instrument TRACE.

At the same time we invited Petr Heinzel to Meudon. After working on prominences and post-flare loops with Jun Elin Wiik (see Section 2.3), he oriented his research towards developing his NLTE radiative-transfer codes to interpret the SUMER spectra. With SUMER we could observe all the hydrogen Lyman series lines and derive the plasma conditions in filaments, prominences and chromospheric fibrils. Lyman lines obtained with SUMER were a good diagnostics for determining the characteristics of the plasma (Wiik et al., 1997, Wiik et al., 1999, Schmieder et al., 1999). Many interesting results were obtained on eruptive prominence, multi-threads and fine threads in prominences (Schmieder et al., 2004c). In particular we showed that filament absorption fine structures observed in $\mathrm{H} \alpha$ with SST corresponded exactly to the darker fine threads observed at $195 \AA$ by TRACE in the filament channel.

Petr Heinzel developed his MALI codes to adapt them to many different cases and structures. It was and it still is a real pleasure to discuss with him. He is a hard worker and always finds a solution. I have always fun to be invited for a barbecue in his place during my visit sin Ondřejov (Figure 14 bottom right panel). We published thirty papers on prominences from 1998 up to now. For ten of them he is the first author, mainly concerning theoretical aspects, for ten other papers on observations I am the first author. The observations are limiting conditions of theoretical models for prominence formation and mass loading for coronal mass ejections during filament eruptions. Let us quote some of them: (Schmieder et al., 1999 Heinzel et al., 2000 Heinzel, Schmieder, and Tziotziou, 2001, Schmieder, Tziotziou, and Heinzel, 2003 Heinzel, Anzer, and Schmieder, 2003: Schmieder et al., 2004b| Schmieder et al., 2007b Heinzel et al., 2008). The other ten papers from the thirty papers were led by scientists of his group in Ondřejov, e.g., Pavel Schwartz, Arek Berlicki, Stano Gunàr, Jaro Dudik (Schwartz et al., 2004; Schwartz et al., 2006, Gunár et al., 2010, Berlicki et al., 2011, Parenti et al., 2012 Gunár et al., 2018). In particular S. Gunàr adapted the MALI 2D-code of radiative transfer to multi-thread structures which could be applied to the SUMER and MSDP observations (Gunár et al., 2007, Gunár et al., 2008 Gunár et al., 2012). The asymmetry in the Lyman line profiles could be explained by multi-structures having different velocities along the line of sight.

With Hinode, a Japanese solar mission, high resolution observations were obtained in the optical range with the SOT. They allowed us to see fine structures of prominences and their high dynamic nature in $\mathrm{Ca}$ II and $\mathrm{H} \alpha$ lines. Many intriguing structures were 

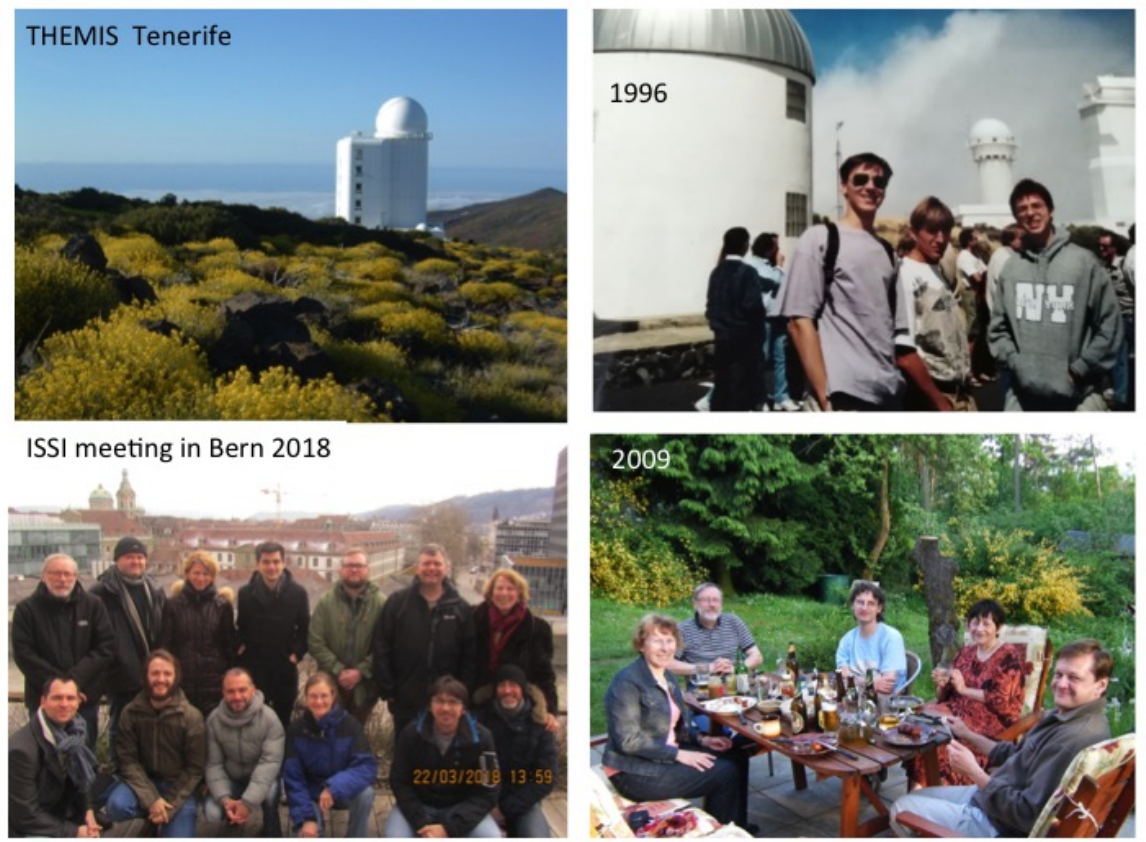

Figure 14. Top left, THEMIS in Tenerife in 2008, top right: visit of the observatory in Ténerife during the EU meeting in 1996: Guillaume Aulanier, Guillaume Molodij, Arturo Lopez Ariste, bottom left, ISSI meeting in Bern on Paradoxes in solar physics: atypical dynamic prominences, first row: Stano Gunár, Jack Jenkins, Manuel Luna, Terry Kucera, Arturo Lopez Ariste, Nicola Labrosse (PI), second row: Petr Heinzel, Maciej Zapiór, Sonja Jejčič, T.Rees-Crockford, Andrew Rodger, Duncan Mackay, BS, bottom right: Dinner in the garden of Petr Heinzel, from left to right BS, Petr Heinzel, Pavol Schwartz, the wife of P.Heinzel, Arek Berlicki in 2009.

discovered, e.g., rising bubbles, plumes, vertical threads. I started to discuss with my MHD group how we could reconcile the MHD model of horizontal field lines with dips developed in the Aulanier's thesis (Aulanier et al., 1998b) with such observations. We invited Jaro Dudik to model these structures and we concluded that the quasi-vertical threads were just pile-up of dips in horizontal magnetic field lines and the bubble was a magnetized volume surrounded by a separatrix and a null point (Dudík et al., 2008, Dudík et al., 2012). We demonstrated that the bubbles were not hotter than the surrounding corona (Gunár et al., 2014). Reconnection at the null point could initiate the dynamics of the plasma and the direction change of the dips to form a plume.

With the new UV spectrograph, Interface Region Imaging Spectrograph (IRIS), launched by NASA in 2013, we continued to organize multi-wavelength observations during coordinated campaign with THEMIS in Tenerife and the MSDP at the Meudon solar tower in the years 2013 - 2016 (Figure 16). I was observing with Arturo Lopez Ariste, then resident astronomer at THEMIS, our French magnetograph in Canary Islands (Figure 14 top panels). It was a great experience for all of our team as they joined us successively (Nicolas Labrosse, Peter Levens (PhD student of Nicolas), Stano Gunár). We observed more than 138 prominences and we confirmed the old results of Véronique Bommier, Sylvie Sahal, and Jean Louis Leroy that the magnetic field in prominences is mainly horizontal (Lopez Ariste, 2014). 


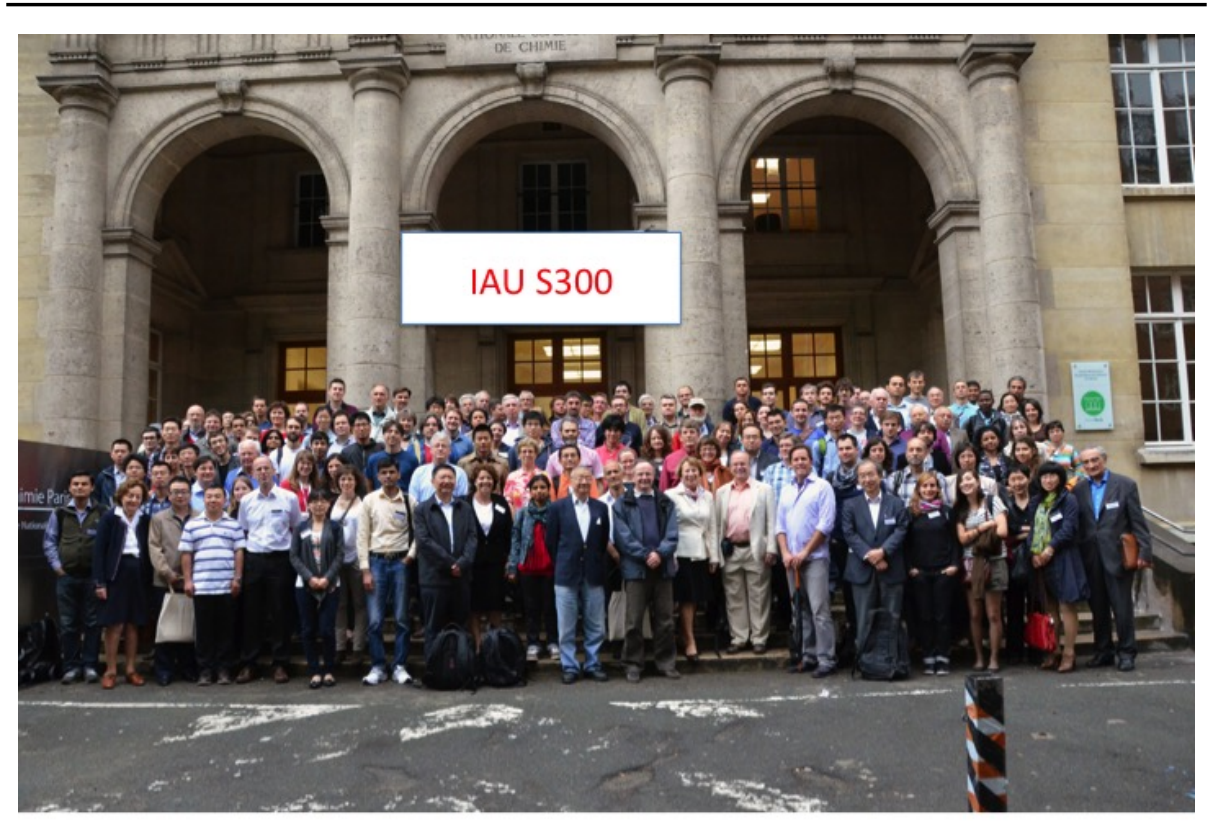

Figure 15. IAU S300 Symposium on the Nature of prominences and their role in Space Weather in Paris in June 2013 in memoriam of Einar Tandberg-Hanssen: the participants in "Ecole de Chimie" in Paris.

The high spatial resolution of IRIS allowed us to measure Dopplershifts. Even in quiescent prominences flows could reach 60 to $70 \mathrm{~km} \mathrm{~s}^{-1}$ (Schmieder et al., 2013 , Schmieder et al., 2014a). The thesis of Peter Levens in Glasgow was based on these campaigns of observation and focused on the existence of "solar tornadoes" (Levens et al., 2016a; Levens et al., 2016b Levens et al., 2017).

Many filaments approaching the limb looked like tornadoes when observed in SDO/AIA movies. They appeared to rotate around their axes like tornadoes on Earth. Therefore they were named tornadoes. However, we found their rotation to be very suspicious and that these tornadoes would be better interpreted as the legs of prominences observed from a certain perspective as they cross the limb as demonstrated by a 3D reconstruction of the magnetic field lines for a helical prominence (Schmieder et al., 2017b Schmieder et al., 2017a). This topic led to press releases at the Observatoire de Paris (1], at IRAP, and at the University of Glasgow in April 2018. In these papers Arturo Lopez Ariste processed the data of THEMIS and concluded that the magnetic field was not vertical as it looks to be in tornadoes. We continued our collaboration with Petr Heinzel working on the Mg II line profiles observed with IRIS (Heinzel et al., 2015). We obtained new data in 2017 with the MSDP at the solar tower jointly with IRIS spectra. These lines give strong constraints on radiative-transfer models in 1D and 2D (Ruan et al., 2018).

${ }^{1}$ https://www.obspm.fr/les-tornades-solaires-ne-sont.html?lang $=\mathrm{fr}$ 


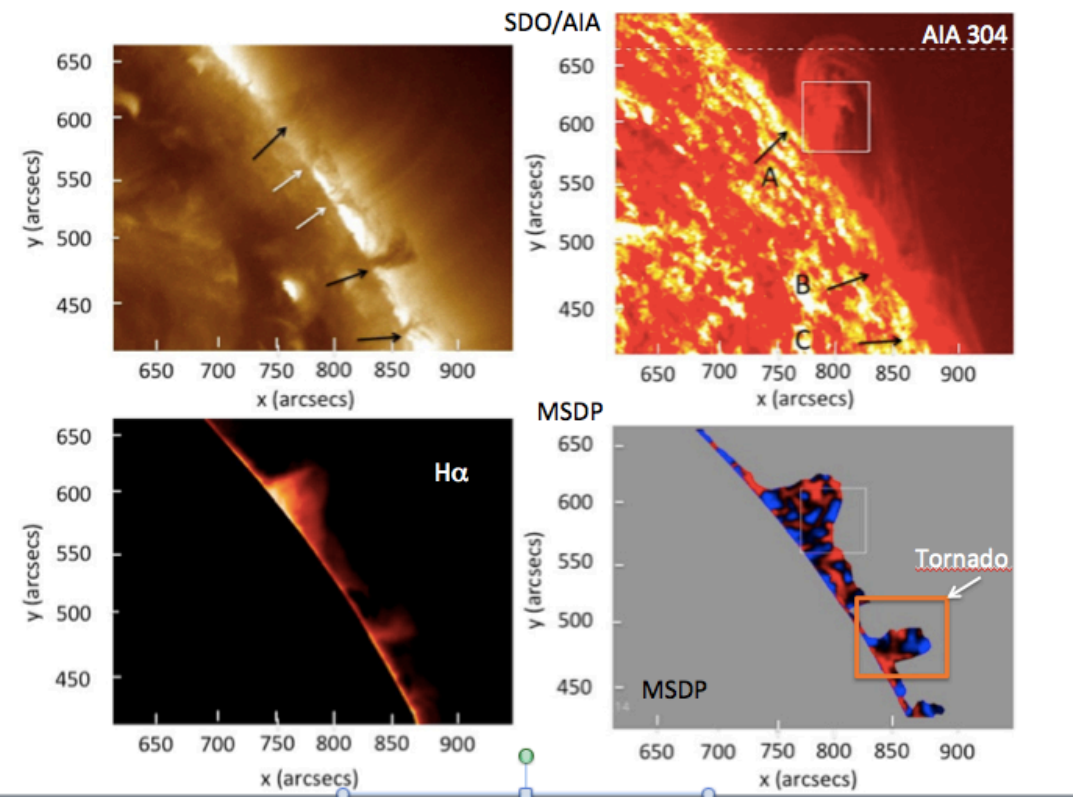

Figure 16. Prominence observed during a campaign of observations with IRIS, top left with SDO/AIA $193 \AA$, top right AIA $304 \AA$, bottom left in $\mathrm{H} \alpha$ with the Meudon solar tower, bottom right the Doppler shift in the prominence and in the tornado (Schmieder et al 2014a, Schmieder et al 2017a).

J.M. Malherbe, S.T. Wu, and myself, organized in Paris an IAU symposium on 'The nature of prominences and their role in space weather", IAU S300, in June 2013, dedicated to Einar Tanberg-Hanssen who died in 2011 (Figure 10 and 15). Together we published a book (Schmieder, Malherbe, and Wu, 2014). After intensive Team Meetings in Bern, at ISSI, we wrote two important reviews on prominences (Labrosse et al., 2010, Mackay et al., 2010). More recently we got a new ISSI Team project on tornadoes (Figure 14 bottom left panel). We are currently writing a review on tornadoes which should be published soon (Labrosse et al 2019, in preparation).

\subsection{Flares and CMEs - THEMIS - 2000 - 2020}

Since SMM we observed flares during all our multi-wavelength campaigns with the MSDP, either at the Meudon solar tower (1980's), or at Pic du Midi (1990's), and finally with THEMIS after 2000 (Figure 14 top left). I spent nearly two weeks per year on these campaigns with Pierre and Nicole Mein. Always a student accompanied us, e.g., Jean-Marie Malherbe, Pascal Démoulin, Guillaume Aulanier, Yang Guo, Petr Levens. It was very exiting to observe at THEMIS on the top of the mountain (Figure 14 top panels). We were accommodated at the summit and benefited from beautiful sunsets. THEMIS, designed by Jean Rayrole with his two post-focal instruments, the MTR and the MSDP, was built well after the first designs were made and finally their promotors died or retired before it was 

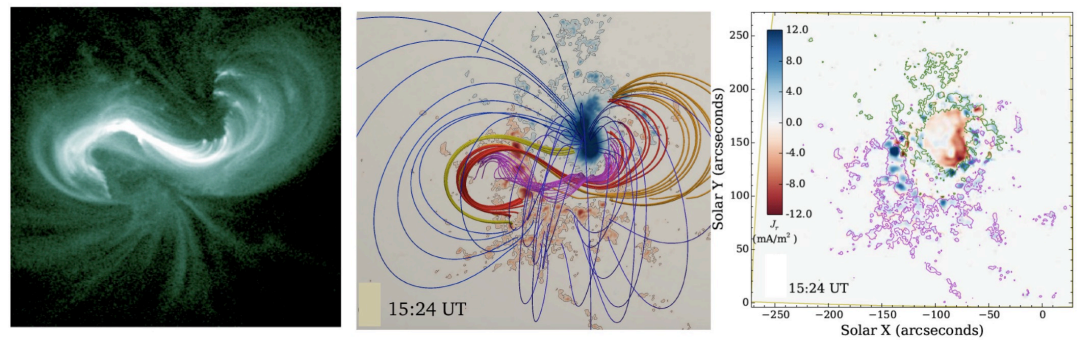

Figure 17. Left, Eruptive sigmoidal loop in the corona observed by SDO/AIA at $94 \AA$, middle: reconstruction of the magnetic loop by a non-linear force free extrapolation, right: electric current measured in the photosphere using SDO/HMI data (Zhao et al 2016).

fully operational. Therefore the young generation never completely invested its effort in the instrument and many of them left the field. The present director of THEMIS, Bernard Gelly, is still convinced of the necessity of THEMIS which is presently the only magnetograph working on the ground, i.e. for prominences. Gelly is working on the installation of adaptive optics for THEMIS which reopens in 2019. We hope that THEMIS will soon get a second life.

Coming back to the end of the 1990's, I had the pleasure to have Guillaume Aulanier as a PhD student. For his thesis (1998) G. Aulanier developed a linear force-free extrapolation code for filament support. Using his code we showed that the differential shear above the inversion line in an active region induced the formation of a filament low down and that the magnetic field progressively becomes more potential with increasing altitude in the corona (Schmieder et al., 1996a). By the way the importance of 3D magnetic configurations to support prominences and to understand their destabilization were fully demonstrated (Schmieder et al., 1999, Kucera et al., 1999, Aulanier and Schmieder, 2002, Dudík et al., 2008; Dudík et al., 2012).

With the observations obtained with Yohkoh and the MSDP at Pic du Midi, G. Aulanier and I worked on flare ribbons and flare loops to understand how reconnection in flares can occur (Schmieder et al., 1997). Later joint THEMIS/MSDP observations with SDO allowed us to investigate the physical conditions of the flaring active regions including their magnetic topology Aulanier, Janvier, and Schmieder, 2012, Dalmasse et al., 2015; Joshi et al., 2016, Joshi et al., 2019; Zhao et al., 2016) (Figure 17). 
In flares the role of bald patch (Schmieder et al., 1997; Aulanier et al., 1998c; López Ariste et al., 2006), null point (Li et al., 2006 Schmieder et al., 2007a), magnetic twist in flux rope (van Driel-Gesztelyi et al., 2000 Canou et al., 2009; Török et al., 2009, Guo et al., 2010| Schmieder et al., 2013), emerging flux (Tang et al., 2000\} Pariat et al., 2004 | Schmieder et al., 2006: Chandra et al., 2009), slipping reconnection (Dudík et al., 2016), flux rope reconnection (Török et al., 2011) was intensively analysed in the group.

After working on so many case-studies, G. Aulanier wrote his own MHD simulation code (the Observationally driven High-order scheme Magnetohydrodynamic code- OHM), which allowed him to develop a 3D extension of the flare standard model (Aulanier, Démoulin, and Grappin, 2005; Aulanier et al., 2010). He found in his 3D models innovative solutions to explain different signatures of the flares, e.g., flare ribbons, post flare loops, vortex Aulanier, Janvier, and Schmieder, 2012, Aulanier et al., 2013, Janvier et al., 2014; Zhao et al., 2016| Zuccarello et al., 2017; Dudík et al., 2017) and the causes of flares-CMEs (Schmieder and Aulanier, 2012). We approached the topic of electric currents, observations and theory by using OHM to see if there is a net current before flares (Schmieder and Aulanier, 2018).

The intuitive sense in physics and fast thinking of G. Aulanier are very important in the group. He is still interested in all observations made on the ground with THEMIS, as well as in space (from Yohkoh to SDO). His high level of knowledge leads him to find unexpected solutions to explain the causes of flares, bright points and eruptions. Therefore he is involved in many collaborations.

Up to now P. D'emoulin, G. Aulanier and me make a very good team to lead $\mathrm{PhD}$ students and post docs in Meudon. I can count nearly 50 papers where we are associated during 20 years. Different observational and theoretical aspects of filaments and flares have been treated. I can quote the $\mathrm{PhD}$ students that we supervised, e.g., Arek Berlicki, Etienne Pariat, Yang Guo, Kelvin Dalmasse on the magnetic topology of flaring active regions. We had and still have today intensive and very productive collaborations with foreign countries, e.g., China (Cheng Fang C., Y. Tang, Li Hui,Yang Guo, Guiping Ruan), India (Gosain Sanjay, Chandra Ramesh, Navin), Argentina (Cristina Mandrini), UK (Lidia van Driel), and the Czech Republic (Petr Heinzel, Jaro Dudik, Stano Gunar, Maciej Zapior) and more recently with individual post docs, e.g., Stuart Gilchrist, Jie Zhao, Miho Janvier and Francesco Zuccarello.

Our ideas on physical mechanisms for flares, eruptions, and CMEs are summarized in three reviews (Schmieder, Démoulin, and Aulanier, 2013; Janvier, Aulanier, and Démoulin, 2015 | Schmieder, Aulanier, and Vršnak, 2015).

\section{Space Weather}

Beyond the corona in the heliosphere the solar wind is blowing with a velocity of more than $400 \mathrm{~km} \mathrm{~s}^{-1}$ where coronal mass ejections and accelerated particles are traveling toward the Earth. The new problem which arises at the beginning of the 21st century is the analysis of their effects on Earth. We have identified 
aurora borealis, disruptions to the electric power grid, disturbances in telecommunications. We should be able to predict them in case of extreme events like those observed on other stars.

My first major steps towards Space Weather research were to organize as JOSO president the two SOLSPA conferences in 2000 and 2001 (see Section 2.2 ) and to be vice president of SCOSTEP from 2007 to 2011. I became vice president of SCOSTEP after serving as IAU representative at the SCOSTEP bureau during ten years (1996-2006). I participated in the organization of several General Assemblies, e.g., Longmont, Berlin, Melbourne and in meetings, like in Hokaido during the S-Ramp meeting for the end of the STEP program where I met Ed Cliver and Dave Webb, specialists of extreme events (Figure 8 left panel).

In 2004 the SCOSTEP bureau asked me to form a group with Bob Vincent to define the future program of SCOSTEP and we proposed the Climate and Space Weather of the Sun Earth System (CAWSES) program which was running for eight years (from 2008 to 2016). I discovered that in France we were really pioneers to have such program on the national level, i.e. the PNST ("Programme National Soleil Terre"). I get an award for my services as SCOSTEP vice president in June 2015.

Working on the organization of the science I was also interested to see by myself the causes of magnetospheric disturbances produced by interplanetary coronal mass ejections (ICMEs). I worked in two groups: a French group gathering scientists of different communities and in Bern (ISSI Teams) a group with Belgian scientists, e.g., Luciano Rodriguez, Stefaan Poedts, Argentinian scientists, e.g., Cristina Mandrini, Sergio Dasso, Hebe Cremades and with the Spanish group of Consuelo Cid. This work was very interesting. It showed to me how difficult is to find the causes of magnetospheric disturbances. With different techniques there is still an uncertainty of more than ten hours to be able to predict the arrival of ICMEs to the Earth (Dasso et al., 2009; Rodriguez et al., 2009, Cid et al., 2012, Cremades et al., 2015| Bocchialini et al., 2018).

Extreme solar events causing strong disturbances on the Earth became a hot topic because the satellite Kepler registered extreme events on stars similar to the Sun as discovered by the Shibata group (Shibata et al., 2013). Could that happen with the Sun? Guillaume Aulanier used his OHM simulation and the historical frames of the Sun since 1909 kept in the Meudon archives to demonstrate that it could not happen with the present Sun (Aulanier et al., 2013: Schmieder, 2018).

\section{Conclusion}

Extensive progress on the understanding of our Sun has been made during the last 50 years. I realized that our group has been pioneers of many new ideas in different domains. Let us quote some of them, the heating of the corona not by the acoustic waves existing in the photosphere, the evaporation in solar flares explaining bright flare ribbons, the dynamics of prominences (counter streaming, multi-threads, tornadoes), the filament eruption mass loading of coronal mass ejections, Ellerman bombs and the sea serpentine flux tube as it is crossing 


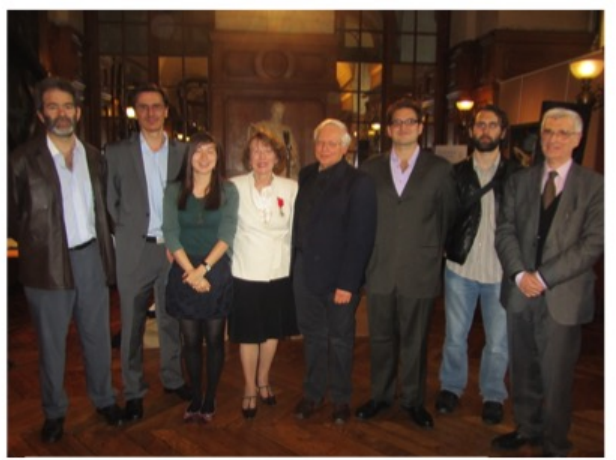

2012 Paris award « Légion d’Honneur »

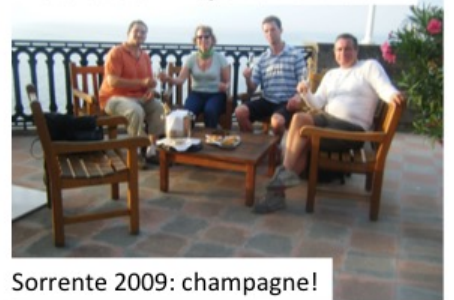

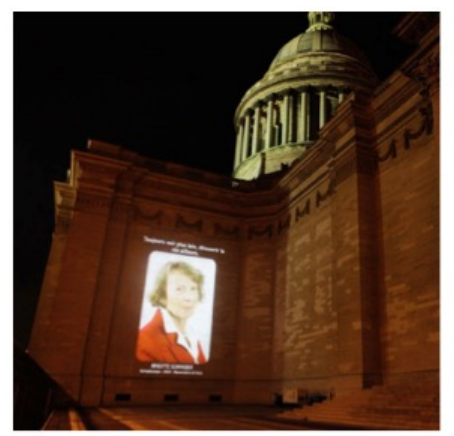

Panthéon 2010

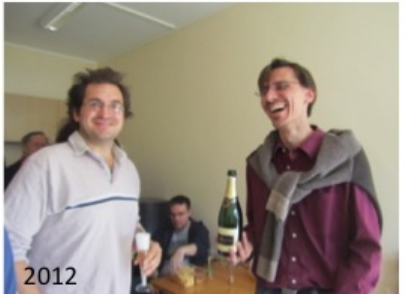

Figure 18. top left, Award to Brigitte Schmieder (BS) (Officier de la Légion d'Honneur) on June 132012 at the Observatoire de Paris given by Jean Claude Pecker. Our solar team: Pascal Démoulin, Guillaume Aulanier, Miho Janvier, BS, Leon Golub, Etienne Pariat, Kevin Dalmasse, Pierre Mein, top right: BS picture projected on the facade of the Pantheon (1000 best researchers in France), bottom left: ESPM meeting in Sorrento 2009, champagne for the CNRS position of Etienne Pariat at the Observatory of Paris, BS, Daniel Muller, Stefaan Poedts, bottom right: champagne again in Meudon with my two former PhD students: Etienne Pariat and Guillaume Aulanier for his HDR thesis.

the solar surface. Our pioneer ideas are often rediscovered ten years later by other groups when the topic becomes fashionable. My list of publications in peer reviews reaches 280 papers today.

This has been possible because of my concept of research including observations and theory. I created a solar MHD group in Meudon with my previous $\mathrm{PhD}$ students. Now we are training many post docs or students from here and abroad. I am personally invited to many countries to give lectures and advice for students. My life was always turned to others: what can I bring to him/her, what can I learn from him or her. It was a continuous exchange and it is why I am traveling to so many countries. During all these years many instruments were developed, in space (SMM, Yohkoh, SOHO, TRACE, Hinode, RHESSI, SDO, IRIS) and on the ground, the French and Italians built a telescope in Tenerife, called THEMIS with a multi-line magnetograph (MTR) and the Multi-Channel Substrative Double Pass (MSDP) spectrograph. Every one of these instruments brought to us new discoveries. THEMIS (1996-2016) was a very successful instrument giving specific results that no other instruments can provide, e.g., measuring the magnetic field in prominences. Now the instrument is upgraded with new adaptive optics, let us hope a new life for it. 

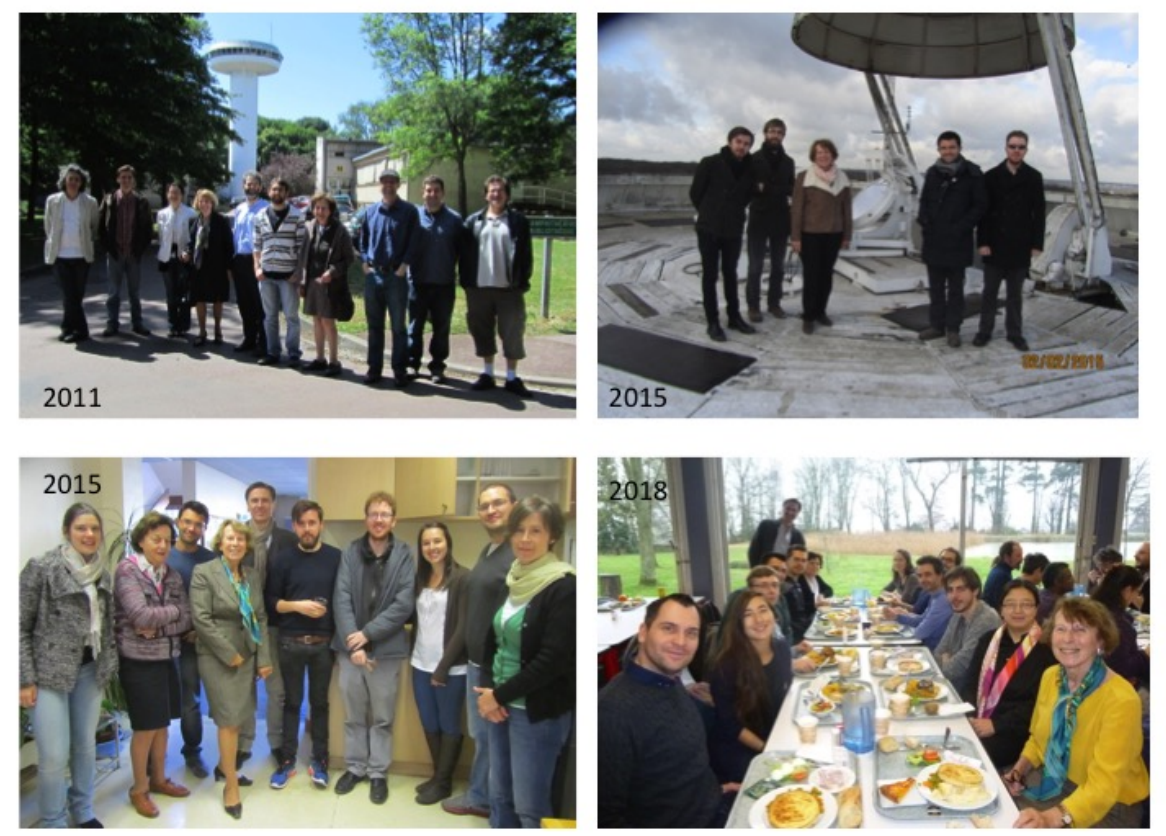

Figure 19. In front of the solar tower of Meudon, the solar group in September 2016: Gehardo Valori, Guillaume Aulanier, Rositsa Miteva, BS, Pascal Démoulin, Kevin Dalmasse, Monique Pick, Marc Linton, Hamish Reid, Etienne Pariat, top right, top of the solar tower with the coelostat in 2015: Eoin Carley, Peter Levens, BS, Francesco Zuccarrello, Stuart Gilchrist, bottom left, in Meudon 2015: Sophie Musset, Monique Pick, F.Zuccarello, BS, G.Aulanier, E. Carley, Stuart Gilchrist, Carolina Malas-Matamoros, Etienne Pariat, Sophie Masson, bottom right, 2018 Meudon cafeteria, from left to right, Stano Gunar, Miho Janvier, Krysztof Barczynski, F.Zuccarello, Jaro Dudik, N.Vilmer, G.Aulanier, S. Masson, E.Pariat, -, Luis Linan, Guiping Ruan, BS.

For the future, in the horizon of the 2020's, we saw already the launch of the Parker Solar Probe to the Sun, we will have soon the departure of Solar Orbiter going also towards the Sun, the first light in the US DKIST solar telescope, and perhaps the construction of EST, the European Solar Telescope, on the ground. I hope that they will bring also important discoveries on the solar wind, its origin and the processes of acceleration of particles. Our solar group should get involved in these instruments.

Nowadays international collaborations are even more important than before because astrophysics is such a complex topic, mixing many different physical processes. The new system of post-docs leaving the group after two or three years increases our potential of research but is not as efficient as collaborations with permanent researchers in other countries who can continue to collaborate.

My career was fantastic and is still very lively. I met a lot of friends and worked in wonderful conditions. I was selected in 2010 among 1000 French scientists to be honored by having projected my photo on the Pantheon during the Science Days in Paris (Figure 18 top right panel). 
I got the awards of "Chevalier and Officier des Palmes académiques" in 2010 and the awards of "Chevalier and Officier de la Légion d'Honneur" in 2012 for my involvement in education and research (Figure 18 top left panel).

I have educated many students (master and $\mathrm{PhD}$ ) and post-docs in solar physics. We celebrated successes with champagne each time (Figure 18 bottom panels). I apologize that I could not quote all of them. I want to thank all my colleagues from the past and those of today. The ambience is still very friendly and I try to communicate my enthusiasm of the research to my younger colleagues from all over the world (Figure 19). During my thesis research, I have to define the problems to resolve. It was a white page on my desk every day and I have to think during all the day what to compute and write. Now I have so many questions in solar physics to answer that I have not enough time and people around me to achieve the task.

I have many other scientific activities as referee of many scientific papers and many proposals (EU, Australian, Belgium FWO). I am member of the SAF committee (Société Astronomique de France) and animated public events such as eclipses, and the passage of Mercury in front of the Sun. I also like dancing, music, listening operas, nature, I like to go to seashore when it is summer time, play the piano, grow flowers and enjoy their beauty.

The salary of astronomer is not so high and it was not easy to travel with kids at home but I thank my family for accepting my life as it was. I have fantastic grandchildren that many of my colleagues know because they accompany me in meetings in different countries (Camille, Adrien, Grégoire, Gautier, Capucine, Baptiste). They are interested in watching the solar eclipses, observing Jupiter and Saturn with a large telescope. The two youngest grandchildren (Clémentine and Margot) hope that it will be soon their turn.

\section{References}

Alissandrakis, C.E., Dialetis, D., Mein, P., Schmieder, B., Simon, G.: 1988, The Evershed flow in the solar photosphere, chromosphere and chromosphere-corona transition region. Astron. Astrophys. 201, 339-349.

Aulanier, G., Démoulin, P.: 1998, 3-D magnetic configurations supporting prominences. I. The natural presence of lateral feet. Astron. Astrophys. 329, 1125-1137.

Aulanier, G., Schmieder, B.: 2002, The magnetic nature of wide EUV filament channels and their role in the mass loading of CMEs. Astron. Astrophys. 386, 1106-1122. doi 10.1051/0004-6361:20020179

Aulanier, G., Démoulin, P., Grappin, R.: 2005, Equilibrium and observational properties of line-tied twisted flux tubes. Astron. Astrophys. 430, 1067-1087. doi 10.1051/00046361:20041519

Aulanier, G., Janvier, M., Schmieder, B.: 2012, The standard flare model in three dimensions. I. Strong-to-weak shear transition in post-flare loops. Astron. Astrophys. 543, A110. doi $10.1051 / 0004-6361 / 201219311$

Aulanier, G., Démoulin, P., van Driel-Gesztelyi, L., Mein, P., Deforest, C.: 1998a, 3-D magnetic configurations supporting prominences. II. The lateral feet as a perturbation of a twisted flux-tube. Astron. Astrophys. 335, 309-322.

Aulanier, G., Démoulin, P., Schmieder, B., Fang, C., Tang, Y.H.: 1998b, Magnetohydrostatic Model of a Bald-Patch Flare. Solar Phys. 183, 369 - 388. doi 10.1023/A:1005003426798 
Aulanier, G., Démoulin, P., Schmieder, B., Fang, C., Tang, Y.H.: 1998c, Magnetohydrostatic Model of a Bald-Patch Flare. Solar Phys. 183, 369-388. doi 10.1023/A:1005003426798

Aulanier, G., Démoulin, P., Mein, N., van Driel-Gesztelyi, L., Mein, P., Schmieder, B.: 1999, 3-D magnetic configurations supporting prominences. III. Evolution of fine structures observed in a filament channel. Astron. Astrophys. 342, $867-880$.

Aulanier, G., Török, T., Démoulin, P., DeLuca, E.E.: 2010, Formation of Torus-Unstable Flux Ropes and Electric Currents in Erupting Sigmoids. Astrophys. J. 708, 314-333. doi $10.1088 / 0004-637 \mathrm{X} / 708 / 1 / 314$

Aulanier, G., Démoulin, P., Schrijver, C.J., Janvier, M., Pariat, E., Schmieder, B.: 2013, The standard flare model in three dimensions. II. Upper limit on solar flare energy. Astron. Astrophys. 549, A66. doi 10.1051/0004-6361/201220406

Berlicki, A., Schmieder, B., Vilmer, N., Aulanier, G., Del Zanna, G.: 2004, Evolution and magnetic topology of the M 1.0 flare of October 22, 2002. Astron. Astrophys. 423, $1119-$ 1131. doi:10.1051/0004-6361:20040259

Berlicki, A., Heinzel, P., Schmieder, B., Mein, P., Mein, N.: 2005, Non-LTE diagnostics of velocity fields during the gradual phase of a solar flare. Astron. Astrophys. 430, 679-689. doi $10.1051 / 0004-6361: 20041293$

Berlicki, A., Gunar, S., Heinzel, P., Schmieder, B., Schwartz, P.: 2011, 2D radiativemagnetohydrostatic model of a prominence observed by Hinode, SoHO/SUMER and Meudon/MSDP. Astron. Astrophys. 530, A143. doi 10.1051/0004-6361/201015896

Bocchialini, K., Grison, B., Menvielle, M., Chambodut, A., Cornilleau-Wehrlin, N., Fontaine, D., Marchaudon, A., Pick, M., Pitout, F., Schmieder, B., Régnier, S., Zouganelis, I.: 2018, Statistical Analysis of Solar Events Associated with Storm Sudden Commencements over One Year of Solar Maximum During Cycle 23: Propagation from the Sun to the Earth and Effects. Solar Phys. 293, 75. doi 10.1007/s11207-018-1278-5

Canou, A., Amari, T., Bommier, V., Schmieder, B., Aulanier, G., Li, H.: 2009, Evidence for a Pre-Eruptive Twisted Flux Rope Using the Themis Vector Magnetograph. Astrophys. J. Lett. 693, L27-L30. doi 10.1088/0004-637X/693/1/L27.

Chandra, R., Schmieder, B., Aulanier, G., Malherbe, J.M.: 2009, Evidence of Magnetic Helicity in Emerging Flux and Associated Flare. Solar Phys. 258, 53-67. doi $10.1007 /$ s11207-0099392-z

Chandra, R., Schmieder, B., Mandrini, C.H., Démoulin, P., Pariat, E., Török, T., Uddin, W.: 2011, Homologous Flares and Magnetic Field Topology in Active Region NOAA 10501 on 20 November 2003. Solar Phys. 269, 83-104. doi 10.1007/s11207-010-9670-9.

Chandra, R., Chen, P.F., Joshi, R., Joshi, B., Schmieder, B.: 2018, Observations of Two Successive EUV Waves and Their Mode Conversion. Astrophys. J. 863, 101. doi $10.3847 / 1538-4357 /$ aad097

Cid, C., Cremades, H., Aran, A., Mandrini, C., Sanahuja, B., Schmieder, B., Menvielle, M., Rodriguez, L., Saiz, E., Cerrato, Y., Dasso, S., Jacobs, C., Lathuillere, C., Zhukov, A.: 2012, Can a halo CME from the limb be geoeffective? Journal of Geophysical Research (Space Physics) 117, A11102. doi 10.1029/2012JA017536.

Cremades, H., Mandrini, C.H., Schmieder, B., Crescitelli, A.M.: 2015, Coronal Mass Ejections from the Same Active Region Cluster: Two Different Perspectives. Solar Phys. 290, 16711686. doi $10.1007 / \mathrm{s} 11207-015-0717-9$

Dalmasse, K., Chandra, R., Schmieder, B., Aulanier, G.: 2015, Can we explain atypical solar flares? Astron. Astrophys. 574, A37. doi 10.1051/0004-6361/201323206

Dalmasse, K., Pariat, É., Valori, G., Jing, J., Démoulin, P.: 2018, Studying the Transfer of Magnetic Helicity in Solar Active Regions with the Connectivity-based Helicity Flux Density Method. Astrophys. J. 852, 141. doi $10.3847 / 1538-4357 /$ aaale1

Dasso, S., Mandrini, C.H., Schmieder, B., Cremades, H., Cid, C., Cerrato, Y., Saiz, E., Démoulin, P., Zhukov, A.N., Rodriguez, L., Aran, A., Menvielle, M., Poedts, S.: 2009, Linking two consecutive nonmerging magnetic clouds with their solar sources. Journal of Geophysical Research (Space Physics) 114, A02109. doi:10.1029/2008JA013102

del Zanna, G., Schmieder, B., Mason, H., Berlicki, A., Bradshaw, S.: 2006, The Gradual Phase of the X17 Flare on October 28, 2003. Solar Phys. 239, 173-191. doi $10.1007 /$ s11207-0060184-4

Delannée, C., Artzner, G., Schmieder, B., Parenti, S.: 2014, Time Evolution of the Altitude of an Observed Coronal Wave. Solar Phys. 289, 2565-2585. doi 10.1007/s11207-014-0488-8.

Démoulin, P., Priest, E.R.: 1988, Instability of a prominence supported in a linear force-free field. Astron. Astrophys. 206, 336-347. 
Démoulin, P., Priest, E.R.: 1993, A model for an inverse-polarity prominence supported in a dip of a quadrupolar region. Solar Phys. 144, 283-305. doi 10.1007/BF00627594.

Démoulin, P., Raadu, M.A.: 1992, Helical structures around quiescent solar prominences computed from observable magnetic fields. Solar Phys. 142, $291-311$. doi $10.1007 /$ BF00151455

Démoulin, P., Malherbe, J.M., Priest, E.R.: 1989, The magnetic field around quiescent solar prominences computed from observational boundary conditions. Astron. Astrophys. 211, $428-440$.

Démoulin, P., Malherbe, J.M., Schmieder, B., Raadu, M.A.: 1987, Fine structures in solar filaments. I - Observations and thermal stability. Astron. Astrophys. 183, 142-150.

Démoulin, P., Henoux, J.C., Priest, E.R., Mandrini, C.H.: 1996, Quasi-Separatrix layers in solar flares. I. Method. Astron. Astrophys. 308, 643-655.

Démoulin, P., Bagala, L.G., Mandrini, C.H., Henoux, J.C., Rovira, M.G.: 1997, Quasiseparatrix layers in solar flares. II. Observed magnetic configurations. Astron. Astrophys. 325, $305-317$

Deng, Y.Y., Schmieder, B., Mandrini, C.H., Khan, J.I., Démoulin, P., Rudawy, P.: 1999, The evolution from birth to decay of a short-lived active region. Astron. Astrophys. 349, $927-940$

Dere, K.P., Schmieder, B., Alissandrakis, C.E.: 1990, Flow patterns in a sunspot region observed in the photosphere, chromosphere and transition region. Astron. Astrophys. 233, $207-219$

Dudík, J., Aulanier, G., Schmieder, B., Bommier, V., Roudier, T.: 2008, Topological Departures from Translational Invariance along a Filament Observed by THEMIS. Solar Phys. 248, 29 - 50. doi $10.1007 /$ s11207-008-9155-2

Dudík, J., Aulanier, G., Schmieder, B., Zapiór, M., Heinzel, P.: 2012, Magnetic Topology of Bubbles in Quiescent Prominences. Astrophys. J. 761, 9. doi $10.1088 / 0004-637 X / 761 / 1 / 9$

Dudík, J., Polito, V., Janvier, M., Mulay, S.M., Karlický, M., Aulanier, G., Del Zanna, G., Dzifč́ková, E., Mason, H.E., Schmieder, B.: 2016, Slipping Magnetic Reconnection, Chromospheric Evaporation, Implosion, and Precursors in the 2014 September 10 X1.6-Class Solar Flare. Astrophys. J. 823, 41. doi 10.3847/0004-637X/823/1/41

Dudík, J., Zuccarello, F.P., Aulanier, G., Schmieder, B., Démoulin, P.: 2017, Expanding and Contracting Coronal Loops as Evidence of Vortex Flows Induced by Solar Eruptions. Astrophys. J. 844, 54. doi 10.3847/1538-4357/aa7aab

Fang, C.: 2018, Sixty-Year Career in Solar Physics. Solar Phys. 293, 72. doi 10.1007/s11207018-1291-8

Fontenla, J., Schmieder, B., Simnett, G.M., Tandberg-Hanssen, E.: 1994, Time evolution of a miniflare as seen in H-alpha, UV lines, and X-rays. Astrophys. J. 424, 1022-1031. doi $10.1086 / 173953$

Forbes, T.G., Malherbe, J.M.: 1986, A shock condensation mechanism for loop prominences. Astrophys. J. Lett. 302, L67-L70. doi 10.1086/184639

Georgoulis, M.K., Rust, D.M., Bernasconi, P.N., Schmieder, B.: 2002, Statistics, Morphology, and Energetics of Ellerman Bombs. Astrophys. J. 575, 506-528. doi 10.1086/341195

Grubecka, M., Schmieder, B., Berlicki, A., Heinzel, P., Dalmasse, K., Mein, P.: 2016, Height formation of bright points observed by IRIS in Mg II line wings during flux emergence. Astron. Astrophys. 593, A32. doi 10.1051/0004-6361/201527358

Gu, X.-M., Lin, J., Luan, T., Schmieder, B.: 1992, Time evolution of a two-ribbon flare Characteristics of post-flare loops. Astron. Astrophys. 259, 649-662.

Gu, X.-M., Ding, Y.-J., Luo, Z., Schmieder, B.: 1997, Post-flare loops of May 16, 1981. Spectral analysis. Astron. Astrophys. 324, 289-297.

Gunár, S., Heinzel, P., Schmieder, B., Schwartz, P., Anzer, U.: 2007, Properties of prominence fine-structure threads derived from SOHO/SUMER hydrogen Lyman lines. Astron. Astrophys. 472, 929-936. doi 10.1051/0004-6361:20077785

Gunár, S., Heinzel, P., Anzer, U., Schmieder, B.: 2008, On Lyman-line asymmetries in quiescent prominences. Astron. Astrophys. 490, 307-313. doi 10.1051/0004-6361:200810127

Gunár, S., Schwartz, P., Schmieder, B., Heinzel, P., Anzer, U.: 2010, Statistical comparison of the observed and synthetic hydrogen Lyman line profiles in solar prominences. Astron. Astrophys. 514, A43. doi 10.1051/0004-6361/200913411

Gunár, S., Mein, P., Schmieder, B., Heinzel, P., Mein, N.: 2012, Dynamics of quiescent prominence fine structures analyzed by $2 \mathrm{D}$ non-LTE modelling of the $\mathrm{H} \alpha$ line. Astron. Astrophys. 543, A93. doi 10.1051/0004-6361/201218940 
Gunár, S., Schwartz, P., Dudík, J., Schmieder, B., Heinzel, P., Jurčák, J.: 2014, Magnetic field and radiative transfer modelling of a quiescent prominence. Astron. Astrophys. 567, A123. doi $10.1051 / 0004-6361 / 201322777$

Gunár, S., Dudík, J., Aulanier, G., Schmieder, B., Heinzel, P.: 2018, Importance of the $\mathrm{H} \alpha$ Visibility and Projection Effects for the Interpretation of Prominence Fine-structure Observations. Astrophys. J. 867, 115. doi 10.3847/1538-4357/aae4e1

Guo, Y., Ding, M.D., Schmieder, B., Li, H., Török, T., Wiegelmann, T.: 2010, Driving Mechanism and Onset Condition of a Confined Eruption. Astrophys. J. Lett. 725, L38-L42. doi $10.1088 / 2041-8205 / 725 / 1 /$ L38

Heinzel, P., Anzer, U., Schmieder, B.: 2003, A Spectroscopic Model of euv Filaments. Solar Phys. 216, 159 - 171. doi 10.1023/A:1026130028966

Heinzel, P., Schmieder, B., Mein, P.: 1992, Structure and dynamics of cool flare loops. Solar Phys. 139, $81-104$. doi $10.1007 /$ BF00147883

Heinzel, P., Schmieder, B., Tziotziou, K.: 2001, Why Are Solar Filaments More Extended in Extreme-Ultraviolet Lines than in H $\alpha$ ? Astrophys. J. Lett. 561, L223-L227. doi $10.1086 / 324755$

Heinzel, P., Schmieder, B., Vial, J.C., Rudawy, P.: 2000, Solar Prominences Observed by Soho (SUMER, CDS, EIT) and Ground-Based Observatories (GBO). Advances in Space Research 25, 1777 -1780. doi 10.1016/S0273-1177(99)00626-2

Heinzel, P., Schmieder, B., Fárník, F., Schwartz, P., Labrosse, N., Kotrč, P., Anzer, U., Molodij, G., Berlicki, A., DeLuca, E.E., Golub, L., Watanabe, T., Berger, T.: 2008, Hinode, TRACE, $\mathrm{SOHO}$, and Ground-based Observations of a Quiescent Prominence. Astrophys. J. 686, 1383 - 1396. doi $10.1086 / 591018$

Heinzel, P., Schmieder, B., Mein, N., Gunár, S.: 2015, Understanding the Mg II and H $\alpha$ Spectra in a Highly Dynamical Solar Prominence. Astrophys. J. Lett. 800, L13. doi $10.1088 / 2041-$ 8205/800/1/L13

Janvier, M., Aulanier, G., Démoulin, P.: 2015, From Coronal Observations to MHD Simulations, the Building Blocks for 3D Models of Solar Flares (Invited Review). Solar Phys. 290, 3425-3456. doi $10.1007 /$ s11207-015-0710-3

Janvier, M., Aulanier, G., Bommier, V., Schmieder, B., Démoulin, P., Pariat, E.: 2014, Electric Currents in Flare Ribbons: Observations and Three-dimensional Standard Model. Astrophys. J. 788, 60. doi 10.1088/0004-637X/788/1/60

Joshi, N.C., Schmieder, B., Magara, T., Guo, Y., Aulanier, G.: 2016, Chain Reconnections Observed in Sympathetic Eruptions. Astrophys. J. 820, 126. doi 10.3847/0004$637 \mathrm{X} / 820 / 2 / 126$

Joshi, N.C., Zhu, X., Schmieder, B., Aulanier, G., Janvier, M., Joshi, B., Magara, T., Chandra, R., Inoue, S.: 2019, Generalization of the Magnetic Field Configuration of Typical and Atypical Confined Flares. Astrophys. J. 871, 165. doi 10.3847/1538-4357/aaf3b5

Kucera, T.A., Aulanier, G., Schmieder, B., Mein, N., Vial, J.-C.: 1999, Filament channel structures in a SI IV line related to a 3d magnetic model. Solar Phys. 186, 259-280. doi $10.1023 / \mathrm{A}: 1005163226096$

Labrosse, N., Heinzel, P., Vial, J.-C., Kucera, T., Parenti, S., Gunár, S., Schmieder, B., Kilper, G.: 2010, Physics of Solar Prominences: I. Spectral Diagnostics and Non-LTE Modelling. Space Sci. Rev. 151, 243 -332. doi 10.1007/s11214-010-9630-6.

Levens, P.J., Schmieder, B., López Ariste, A., Labrosse, N., Dalmasse, K., Gelly, B.: 2016a, Magnetic Field in Atypical Prominence Structures: Bubble, Tornado, and Eruption. Astrophys. J. 826, 164. doi 10.3847/0004-637X/826/2/164

Levens, P.J., Schmieder, B., Labrosse, N., López Ariste, A.: 2016b, Structure of Prominence Legs: Plasma and Magnetic Field. Astrophys. J. 818, 31. doi 10.3847/0004$637 \mathrm{X} / 818 / 1 / 31$

Levens, P.J., Labrosse, N., Schmieder, B., López Ariste, A., Fletcher, L.: 2017, Comparing $\mathrm{UV} / \mathrm{EUV}$ line parameters and magnetic field in a quiescent prominence with tornadoes. Astron. Astrophys. 607, A16. doi 10.1051/0004-6361/201730808

Li, H., Schmieder, B., Aulanier, G., Berlicki, A.: 2006, Is Pre-Eruptive Null Point Reconnection Required for Triggering Eruptions? Solar Phys. 237, 85-100. doi $10.1007 /$ s11207-0060173-7.

Lopez Ariste, A.L.: 2014, Instrument concepts for the observation of prominences with future ground-based telescopes. In: Schmieder, B., Malherbe, J.-M., Wu, S.T. (eds.) Nature of Prominences and their Role in Space Weather, IAU Symposium 300, 370-375. doi $10.1017 /$ S1743921313011216 
López Ariste, A., Aulanier, G., Schmieder, B., Sainz Dalda, A.: 2006, First observation of bald patches in a filament channel and at a barb endpoint. Astron. Astrophys. 456, 725-735. doi 10.1051/0004-6361:20064923

Mackay, D.H., Karpen, J.T., Ballester, J.L., Schmieder, B., Aulanier, G.: 2010, Physics of Solar Prominences: II. Magnetic Structure and Dynamics. Space Sci. Rev. 151, 333-399. doi $10.1007 / \mathrm{s} 11214-010-9628-0$

Malherbe, J.M., Schmieder, B., Mein, P.: 1981, Dynamics in the filaments. I - Oscillations in a quiescent filament. Astron. Astrophys. 102, 124-128.

Malherbe, J.M., Simon, G., Mein, P., Mein, N., Schmieder, B., Vial, J.C.: 1982, Preflare heating of filaments. Advances in Space Research 2, 53-56. doi 10.1016/0273-1177(82)90178-8

Malherbe, J.M., Schmieder, B., Ribes, E., Mein, P.: 1983, Dynamics of solar filaments. II - Mass motions in an active region filament from $\mathrm{H}$-alpha center to limb observations. Astron. Astrophys. 119, $197-206$.

Malherbe, J.M., Schmieder, B., Simon, G., Mein, P., Tandberg-Hanssen, E.: 1987, Closed magnetic structures in the chromosphere and in the transition region. Solar Phys. 112, 233 - 253. doi $10.1007 /$ BF00148780

Malherbe, J.-M., Tarbell, T., Wiik, J.E., Schmieder, B., Frank, Z., Shine, R.A., van DrielGesztelyi, L.: 1997, The Postflare Loops and the Nearby Active Chromosphere of 1992 June 26. Astrophys. J. 482, 535-540. doi 10.1086/304141

Mandrini, C.H., Démoulin, P., van Driel-Gesztelyi, L., Schmieder, B., Cauzzi, G., Hofmann, A.: 1996, 3D Magnetic Reconnection at an X-Ray Bright Point. Solar Phys. 168, 115-133. doi $10.1007 /$ BF00145829

Mandrini, C.H., Démoulin, P., Bagala, L.G., van Driel-Gesztelyi, L., Henoux, J.C., Schmieder, B., Rovira, M.G.: 1997, Evidence of Magnetic Reconnection from H $\alpha$, Soft X-Ray and Photospheric Magnetic Field Observations. Solar Phys. 174, 229-240. doi $10.1023 / \mathrm{A}: 1004950009970$

Mandrini, C.H., Démoulin, P., Schmieder, B., Deluca, E.E., Pariat, E., Uddin, W.: 2006, Companion Event and Precursor of the X17 Flare on 28 October 2003. Solar Phys. 238, 293 - 312. doi $10.1007 /$ s11207-006-0205-3

Mandrini, C.H., Schmieder, B., Démoulin, P., Guo, Y., Cristiani, G.D.: 2014, Topological Analysis of Emerging Bipole Clusters Producing Violent Solar Events. Solar Phys. 289, 2041 - 2071. doi 10.1007/s11207-013-0458-6.

Martres, M.-J., Mein, P., Schmieder, B., Soru-Escaut, I.: 1981, Structure and evolution of velocities in quiescent filaments. Solar Phys. 69, 301-312. doi 10.1007/BF00149996

Mein, N., Schmieder, B.: 1981, Mechanical flux in the solar chromosphere. III - Variation of the mechanical flux. Astron. Astrophys. 97, 310-316.

Mein, N., Schmieder, B., Simon, G., Tandberg-Hanssen, E., Wu, S.T.: 1982, Dynamics of the eruptive prominence of 6 May 1980 and its relationship to the coronal transient. Astron. Astrophys. 114, $192-199$.

Moore, R.L., Schmieder, B., Hathaway, D.H., Tarbell, T.D.: 1997, 3-D Magnetic Field Configuration Late in a Large Two-Ribbon Flare. Solar Phys. 176, 153-169. doi $10.1023 / A: 1004990817154$

Parenti, S., Schmieder, B., Heinzel, P., Golub, L.: 2012, On the Nature of Prominence Emission Observed by SDO/AIA. Astrophys. J. 754, 66. doi 10.1088/0004-637X/754/1/66

Pariat, E., Aulanier, G., Schmieder, B., Georgoulis, M.K., Rust, D.M., Bernasconi, P.N.: 2004, Resistive Emergence of Undulatory Flux Tubes. Astrophys. J. 614, 1099-1112. doi $10.1086 / 423891$

Pariat, E., Schmieder, B., Berlicki, A., Deng, Y., Mein, N., López Ariste, A., Wang, S.: 2007, Spectrophotometric analysis of Ellerman bombs in the Ca II, H $\alpha$, and UV range. Astron. Astrophys. 473, 279-289. doi 10.1051/0004-6361:20067011

Priest, E.R.: 2014, A Life of Fun Playing with Solar Magnetic Fields (Special Historical Review). Solar Phys. 289, 3579-3615. doi 10.1007/s11207-014-0554-2

Raadu, M.A., Malherbe, J.M., Schmieder, B., Mein, P.: 1987a, Material ejecta in a disturbed solar filament. Solar Phys. 109, 59-79. doi 10.1007/BF00167399.

Raadu, M.A., Schmieder, B., Mein, N., Gesztelyi, L.: 1987b, Photospheric Induced Destabilization and Ejection of Prominence Material. Hvar Observatory Bulletin 11, 105.

Raadu, M.A., Schmieder, B., Mein, N., Gesztelyi, L.: 1988, Photospheric-induced destabilization and ejection of prominence material. Astron. Astrophys. 197, 289-296.

Rodriguez, L., Zhukov, A.N., Cid, C., Cerrato, Y., Saiz, E., Cremades, H., Dasso, S., Menvielle, M., Aran, A., Mandrini, C., Poedts, S., Schmieder, B.: 2009, Three frontside full halo 
coronal mass ejections with a nontypical geomagnetic response. Space Weather 7, S06003. doi $10.1029 / 2008 S W 000453$.

Roudier, T., Svanda, M., Meunier, N., Keil, S., Rieutord, M., Malherbe, J.M., Rondi, S., Molodij, G., Bommier, V., Schmieder, B.: 2008, Large-scale horizontal flows in the solar photosphere. III. Effects on filament destabilization. Astron. Astrophys. 480, 255-263. doi $10.1051 / 0004-6361: 20077973$

Roudier, T., Schmieder, B., Filippov, B., Chandra, R., Malherbe, J.M.: 2018, Horizontal photospheric flows trigger a filament eruption. Astron. Astrophys. 618, A43. doi $10.1051 / 0004-6361 / 201832937$

Ruan, G., Schmieder, B., Mein, P., Mein, N., Labrosse, N., Gunár, S., Chen, Y.: 2018, On the Dynamic Nature of a Quiescent Prominence Observed by IRIS and MSDP Spectrographs. Astrophys. J. 865, 123. doi 10.3847/1538-4357/aada08

Schmieder, B.: 1972, Fluctuations of Temperature and Density in the Photosphere. Astron. Astrophys. 16, 44.

Schmieder, B.: 1976, Wave propagation in the photosphere. Solar Phys. 47, 435-460. doi $10.1007 /$ BF00154756

Schmieder, B.: 1977, Linear hydrodynamical equations coupled with radiative transfer in a nonisothermal atmosphere. I - Method. Solar Phys. 54, 269-288. doi 10.1007/BF00159919

Schmieder, B.: 1978, Linear hydrodynamical equations coupled with radiative transfer in a nonisothermal atmosphere. II - Application to solar photospheric observations. Solar Phys. 57, 245 - 253. doi $10.1007 /$ BF00160098

Schmieder, B.: 1979, Waves in the low solar chromosphere. Astron. Astrophys. 74, 273-279.

Schmieder, B.: 2018, Extreme solar storms based on solar magnetic field. Journal of Atmospheric and Solar-Terrestrial Physics 180, 46-51. doi 10.1016/j.jastp.2017.07.018.

Schmieder, B., Aulanier, G.: 2012, What are the physical mechanisms of eruptions and CMEs? Advances in Space Research 49, 1598-1606. doi 10.1016/j.asr.2011.10.023

Schmieder, B., Aulanier, G.: 2018, Solar Active Region Electric Currents Before and During Eruptive Flares. In: Keiling, A., Marghitu, O., Wheatland, M. (eds.) Electric Currents in Geospace and Beyond, Washington DC American Geophysical Union Geophysical Monograph Series 235, 391-406. doi 10.1002/9781119324522.ch23

Schmieder, B., Mein, N.: 1980, Mechanical flux in the solar chromosphere. II - Determination of the mechanical flux. Astron. Astrophys. 84, 99-105.

Schmieder, B., Archontis, V., Pariat, E.: 2014, Magnetic Flux Emergence Along the Solar Cycle. Space Sci. Rev. 186, 227 - 250. doi 10.1007/s11214-014-0088-9

Schmieder, B., Aulanier, G., Vršnak, B.: 2015, Flare-CME Models: An Observational Perspective (Invited Review). Solar Phys. 290, 3457 - 3486. doi 10.1007/s11207-015-0712-1

Schmieder, B., Démoulin, P., Aulanier, G.: 2013, Solar filament eruptions and their physical role in triggering coronal mass ejections. Advances in Space Research 51, 1967-1980. doi 10.1016/j.asr.2012.12.026

Schmieder, B., Fang, C., Harra-Murnion, L.K.: 1998, Spectral Analysis of a Thermal Flare on 27 October 1993. Solar Phys. 182, 447 - 457. doi:10.1023/A:1005040821593

Schmieder, B., Golub, L., Antiochos, S.K.: 1994, Comparison between cool and hot plasma behaviors of surges. Astrophys. J. 425, 326-330. doi 10.1086/173987

Schmieder, B., Malherbe, J.-M., Wu, S.T. (eds.): 2014, Nature of Prominences and their role in Space Weather, IAU Symposium 300.

Schmieder, B., Raadu, M.A., Wiik, J.E.: 1991, Fine structure of solar filaments. II - Dynamics of threads and footpoints. Astron. Astrophys. 252, 353-365.

Schmieder, B., Tziotziou, K., Heinzel, P.: 2003, Spectroscopic diagnostics of an $\mathrm{H} \alpha$ and EUV filament observed with THEMIS and SOHO. Astron. Astrophys. 401, 361-375. doi $10.1051 / 0004-6361: 20030126$

Schmieder, B., Mein, P., Vial, J.C., Tandberg-Hanssen, E.: 1982, A surge observed in H alpha and C IV. Advances in Space Research 2, 225-228. doi 10.1016/0273-1177(82)90203-4

Schmieder, B., Mein, P., Vial, J.-C., Tandberg-Hanssen, E.: 1983, Dynamics of a surge observed in the $\mathrm{C}$ IV and $\mathrm{H}$ alpha lines. Astron. Astrophys. 127, 337-344.

Schmieder, B., Simon, G., Martres, M.-J., Mein, P., Mein, N., Tandberg-Hanssen, E.: 1984, Recurrent mass ejections observed in H-alpha and CIV. Advances in Space Research 4, 27 - 30. doi 10.1016/0273-1177(84)90154-6

Schmieder, B., Malherbe, J.M., Simon, G., Poland, A.I.: 1985, Dynamics of solar filaments. IV - Structure and mass flow of an active region filament. Astron. Astrophys. 153, 64-70. 
Schmieder, B., Mein, P., Simnett, G.M., Tandberg-Hanssen, E.: 1988a, An example of the association of X-ray and UV emission with H-alpha surges. Astron. Astrophys. 201, $327-$ 338 .

Schmieder, B., Mein, P., Malherbe, J.-M., Forbes, T.G.: 1988b, Post-flare loops - Formation and velocity. Advances in Space Research 8, 145-148. doi 10.1016/0273-1177(88)90309-2

Schmieder, B., Démoulin, P., Poland, A., Thompson, B.: 1988c, Some dynamical aspects of a quiescent filament. Astron. Astrophys. 197, 281-288.

Schmieder, B., Malherbe, J.M., Simnett, G.M., Forbes, T.G., Tandberg-Hanssen, E.: 1990, Evaporation in the transition region during the gradual phase of flares. Astrophys. J. 356, 720 - 732. doi $10.1086 / 168879$

Schmieder, B., van Driel-Gesztelyi, L., Gerlei, O., Simnett, G.M.: 1993, A study of surges and flares within an active region. Solar Phys. 146, 163-176. doi 10.1007/BF00662177

Schmieder, B., Shibata, K., van Driel-Gesztelyi, L., Freeland, S.: 1995a, H alpha surges and associated soft X-ray loops. Solar Phys. 156, 245-264. doi 10.1007/BF00670226

Schmieder, B., Heinzel, P., Wiik, J.E., Lemen, J., Anwar, B., Kotrc, P., Hiei, E.: 1995b, Relation between cool and hot post-flare loops of 26 June 1992 derived from optical and X-ray (SXT-Yohkoh) observations. Solar Phys. 156, 337-361. doi 10.1007/BF00670231

Schmieder, B., Démoulin, P., Aulanier, G., Golub, L.: 1996a, Differential Magnetic Field Shear in an Active Region. Astrophys. J. 467, 881. doi 10.1086/177662

Schmieder, B., Heinzel, P., van Driel-Gesztelyi, L., Lemen, J.R.: 1996b, Post-Flare Loops of 26 June 1992, II. Solar Phys. 165, 303-328. doi 10.1007/BF00149716

Schmieder, B., Rovira, M., Simnett, G.M., Fontenla, J.M., Tandberg-Hanssen, E.: 1996c, Subflares and surges in AR 2744 during the Solar Maximum Mission. Astron. Astrophys. 308, $957-969$.

Schmieder, B., Aulanier, G., Démoulin, P., van Driel-Gesztelyi, L., Roudier, T., Nitta, N., Cauzzi, G.: 1997, Magnetic reconnection driven by emergence of sheared magnetic field. Astron. Astrophys. 325, 1213-1225.

Schmieder, B., Heinzel, P., Vial, J.C., Rudawy, P.: 1999, SOHO/SUMER observations and analysis of hydrogen Lyman lines in a quiescent prominence. Solar Phys. 189, 109-127. doi $10.1023 /$ A:1005233906192

Schmieder, B., Rust, D.M., Georgoulis, M.K., Démoulin, P., Bernasconi, P.N.: 2004a, Emerging Flux and the Heating of Coronal Loops. Astrophys. J. 601, 530-545. doi $10.1086 / 380199$.

Schmieder, B., Mein, N., Deng, Y., Dumitrache, C., Malherbe, J.-M., Staiger, J., Deluca, E.E.: 2004b, Magnetic changes observed in the formation of two filaments in a complex active region: TRACE and MSDP observations. Solar Phys. 223, 119-141. doi 10.1007/s11207004-1107-X

Schmieder, B., Lin, Y., Heinzel, P., Schwartz, P.: 2004c, Multi-wavelength study of a high-latitude EUV filament. Solar Phys. 221, 297-323. doi $10.1023 /$ B:SOLA.0000035059.50427.68.

Schmieder, B., Aulanier, G., Mein, P., López Ariste, A.: 2006, Evolving Photospheric Flux Concentrations and Filament Dynamic Changes. Solar Phys. 238, 245-259. doi $10.1007 /$ s11207-006-0252-9

Schmieder, B., Gunár, S., Heinzel, P., Anzer, U.: 2007a, Spectral Diagnostics of the Magnetic Field Orientation in a Prominence Observed with SOHO/SUMER. Solar Phys. 241, 5366. doi 10.1007/s11207-007-0251-5

Schmieder, B., Mandrini, C.H., Démoulin, P., Aulanier, G., Li, H., Berlicki, A.: 2007b, What is the role of magnetic null points in large flares? Advances in Space Research 39, 1840-1846. doi $10.1016 /$ j.asr.2007.03.058

Schmieder, B., Guo, Y., Moreno-Insertis, F., Aulanier, G., Yelles Chaouche, L., Nishizuka, N., Harra, L.K., Thalmann, J.K., Vargas Dominguez, S., Liu, Y.: 2013, Twisting solar coronal jet launched at the boundary of an active region. Astron. Astrophys. 559, A1. doi $10.1051 / 0004-6361 / 201322181$

Schmieder, B., Tian, H., Kucera, T., López Ariste, A., Mein, N., Mein, P., Dalmasse, K., Golub, L.: 2014a, Open questions on prominences from coordinated observations by IRIS, Hinode, SDO/AIA, THEMIS, and the Meudon/MSDP. Astron. Astrophys. 569, A85. doi $10.1051 / 0004-6361 / 201423922$

Schmieder, B., Roudier, T., Mein, N., Mein, P., Malherbe, J.M., Chandra, R.: 2014b, Proper horizontal photospheric flows in a filament channel. Astron. Astrophys. 564, A104. doi $10.1051 / 0004-6361 / 201322861$ 
Schmieder, B., Mein, P., Mein, N., Levens, P.J., Labrosse, N., Ofman, L.: 2017a, H $\alpha$ Doppler shifts in a tornado in the solar corona. Astron. Astrophys. 597, A109. doi 10.1051/0004$6361 / 201628771$

Schmieder, B., Zapiór, M., López Ariste, A., Levens, P., Labrosse, N., Gravet, R.: 2017b, Reconstruction of a helical prominence in 3D from IRIS spectra and images. Astron. Astrophys. 606, A30. doi 10.1051/0004-6361/201730839

Schwartz, P., Heinzel, P., Anzer, U., Schmieder, B.: 2004, Determination of the 3D structure of an EUV-filament observed by SoHO/CDS, SoHO/SUMER and VTT/MSDP. Astron. Astrophys. 421, 323-338. doi 10.1051/0004-6361:20034199

Schwartz, P., Heinzel, P., Schmieder, B., Anzer, U.: 2006, Study of an extended EUV filament using SoHO/SUMER observations of the hydrogen Lyman lines. Astron. Astrophys. 459, 651 - 661. doi 10.1051/0004-6361:20065619

She, Z.S., Malherbe, J.M., Raadu, M.A.: 1986, Transition zone effects on thermal nonequilibrium and plasma condensation in solar coronal loops. Astron. Astrophys. 164, $364-372$.

Shibata, K., Isobe, H., Hillier, A., Choudhuri, A.R., Maehara, H., Ishii, T.T., Shibayama, T., Notsu, S., Notsu, Y., Nagao, T., Honda, S., Nogami, D.: 2013, Can Superflares Occur on Our Sun? Pub. Astron. Soc. Japan 65, 49. doi 10.1093/pasj/65.3.49

Simon, G., Schmieder, B., Démoulin, P., Poland, A.I.: 1986, Dynamics of solar filaments. VI - Center-to-limb study of H-alpha and C IV velocities in a quiescent filament. Astron. Astrophys. 166, $319-325$.

Tandberg-Hanssen, E.: 1995, Books-Received - the Nature of Solar Prominences. Science 269, 111.

Tandberg-Hanssen, E.: 2011, Solar Prominences - An Intriguing Phenomenon. Solar Phys. 269, $237-251$. doi $10.1007 /$ s11207-010-9704-3

Tang, Y.H., Li, Y.N., Fang, C., Aulanier, G., Schmieder, B., Démoulin, P., Sakurai, T.: 2000, $\mathrm{H} \alpha$ and Soft X-Ray Brightening Events Caused by Emerging Flux. Astrophys. J. 534, $482-489$. doi $10.1086 / 308715$

Thompson, W.T., Schmieder, B.: 1991, Oscillations in H-alpha filaments - Center-to-limb study. Astron. Astrophys. 243, 501-511.

Török, T., Aulanier, G., Schmieder, B., Reeves, K.K., Golub, L.: 2009, Fan-Spine Topology Formation Through Two-Step Reconnection Driven by Twisted Flux Emergence. Astrophys. J. 704, 485-495. doi 10.1088/0004-637X/704/1/485

Török, T., Chandra, R., Pariat, E., Démoulin, P., Schmieder, B., Aulanier, G., Linton, M.G., Mandrini, C.H.: 2011, Filament Interaction Modeled by Flux Rope Reconnection. Astrophys. J. 728, 65. doi 10.1088/0004-637X/728/1/65

Tsiropoula, G., Schmieder, B.: 1997, Determination of physical parameters in dark mottles. Astron. Astrophys. 324, 1183-1189.

Tsiropoula, G., Alissandrakis, C.E., Schmieder, B.: 1993, The fine structure of a chromospheric rosette. Astron. Astrophys. 271, 574.

Tsiropoula, G., Alissandrakis, C.E., Schmieder, B.: 1994, Time evolution of fine structures in the solar chromosphere. Astron. Astrophys. 290, 285-294.

van Driel-Gesztelyi, L., Schmieder, B., Cauzzi, G., Mein, N., Hofmann, A., Nitta, N., Kurokawa, H., Mein, P., Staiger, J.: 1996, X-Ray Bright Point Flares Due to Magnetic Reconnection. Solar Phys. 163, $145-170$. doi 10.1007/BF00165462

van Driel-Gesztelyi, L., Csepura, G., Schmieder, B., Malherbe, J.-M., Metcalf, T.: 1997, Evolution of a Delta Group in the Photosphere and Corona. Solar Phys. 172, 151-160. doi 10.1023/A:1004975212949

van Driel-Gesztelyi, L., Manoharan, P.K., Démoulin, P., Aulanier, G., Mandrini, C.H., LopezFuentes, M., Schmieder, B., Orlando, S., Thompson, B., Plunkett, S.: 2000, Initiation of CMEs: the role of magnetic twist. Journal of Atmospheric and Solar-Terrestrial Physics 62, 1437 - 1448. doi $10.1016 /$ S1364-6826(00)00087-0

Wiik, J.E., Dere, K., Schmieder, B.: 1993, UV prominences observed with the HRTS: structure and physical properties. Astron. Astrophys. 273, 267.

Wiik, J.E., Heinzel, P., Schmieder, B.: 1992, Determination of plasma parameters in a quiescent prominence. Astron. Astrophys. 260, 419-430.

Wiik, J.E., Schmieder, B., Heinzel, P., Roudier, T.: 1996, Post-Flare Loops of 26 June 1992, III. Solar Phys. 166, $89-106$. doi 10.1007/BF00179357

Wiik, J.E., van Driel-Gesztely, L., Schmieder, B., Heinzel, P.: 1997, Dynamics and evolution of the post-flare loops of June 1992. Advances in Space Research 20, 2345-2349. doi $10.1016 /$ S0273-1177(97)00907-1 
Wiik, J.E., Dammasch, I.E., Schmieder, B., Wilhelm, K.: 1999, Multiple-Thread Model of a Prominence Observed by SUMER and EIT on SOHO. Solar Phys. 187, 405-426. doi $10.1023 /$ A: 1005151015043

Young, P.R., Tian, H., Peter, H., Rutten, R.J., Nelson, C.J., Huang, Z., Schmieder, B., Vissers, G.J.M., Toriumi, S., Rouppe van der Voort, L.H.M., Madjarska, M.S., Danilovic, S., Berlicki, A., Chitta, L.P., Cheung, M.C.M., Madsen, C., Reardon, K.P., Katsukawa, Y., Heinzel, P.: 2018, Solar Ultraviolet Bursts. Space Sci. Rev. 214, 120. doi 10.1007/s11214018-0551-0

Zhao, J., Li, H., Pariat, E., Schmieder, B., Guo, Y., Wiegelmann, T.: 2014, Temporal Evolution of the Magnetic Topology of the NOAA Active Region 11158. Astrophys. J. 787, 88. doi $10.1088 / 0004-637 \mathrm{X} / 787 / 1 / 88$

Zhao, J., Gilchrist, S.A., Aulanier, G., Schmieder, B., Pariat, E., Li, H.: 2016, Hooked Flare Ribbons and Flux-rope-related QSL Footprints. Astrophys. J. 823, 62. doi 10.3847/0004$637 \mathrm{X} / 823 / 1 / 62$

Zhao, J., Schmieder, B., Li, H., Pariat, E., Zhu, X., Feng, L., Grubecka, M.: 2017, Observational Evidence of Magnetic Reconnection for Brightenings and Transition Region Arcades in IRIS Observations. Astrophys. J. 836, 52. doi $10.3847 / 1538-4357 / 836 / 1 / 52$

Zuccarello, F.P., Chandra, R., Schmieder, B., Aulanier, G., Joshi, R.: 2017, Transition from eruptive to confined flares in the same active region. Astron. Astrophys. 601, A26. doi $10.1051 / 0004-6361 / 201629836$ 